\title{
Insulin receptor activation in the nucleus accumbens reflects nutritive value of a recently ingested meal
}

\author{
Woods, C. A. ${ }^{a}$, Guttman, Z. R. ${ }^{a}$, Huang, D. ${ }^{b}$, Kolaric, R.A. ${ }^{b}$, Rabinowitsch, A. I. ${ }^{b}$, Jones, K. T. ${ }^{b}$, \\ Cabeza de Vaca, S. ${ }^{b}$, Sclafani, A. ${ }^{d}$, \& Carr, K.D. ${ }^{a, b, c, e}$ \\ ${ }^{a}$ Center for Neural Science, NYU Graduate School of Arts \& Sciences \\ ${ }^{\mathrm{b}}$ Dept of Psychiatry, NYU School of Medicine \\ ${ }^{\mathrm{C} D e p t}$ of Biochemistry and Molecular Pharmacology, NYU School of Medicine \\ ${ }^{\mathrm{d} D e p t}$ of Psychology, Brooklyn College, CUNY \\ ${ }^{\mathrm{e}}$ Correspondence
}

\begin{abstract}
With respect to feeding, insulin is typically thought of as a satiety hormone, acting in the hypothalamus to limit ingestive behavior. However, accumulating evidence suggests that insulin also has the ability to alter dopamine release in the striatum and influence food preferences. With increased access to high calorie foods, Western societies have a high prevalence of obesity, accompanied by insulin insensitivity. Little is known about how insulin is trafficked into the brain following food consumption and whether insulin insensitivity in the periphery is mirrored in the central nervous system. We investigated insulin receptor activation in the ventral striatum of rats receiving water or $16 \%$ glucose either orally or intragastrically. We also investigated whether glucose-induced insulin receptor activation was altered in food-restricted (FR) or diet-induced obesity (OB) rat models. Lastly, we examined whether insulin plays a significant role in flavor-nutrient preference learning. Glucose intake stimulated a rapid increase in insulin receptor activity in the ventral striatum of $F R$ and ad libitum (AL) fed rats, but not OB rats. Similarly, both $A L$ and FR, but not OB rats demonstrated significant flavor-nutrient preferences. However AL rats receiving brief inhibition of insulin activity during conditioning failed to acquire a significant flavor-nutrient preference. These findings suggest that impaired insulin receptor activation in the ventral striatum may result in inaccurate valuation of nutritive foods, which could lead to overconsumption of food or the selection of foods that don't accurately meet the body's current physiological needs.
\end{abstract}

\section{Introduction}

In addition to serving as a satiety signal in the hypothalamus, recent evidence indicates that insulin acts in the nucleus accumbens (NAc) to enhance dopamine release and communicate the nutritive value of a consumed sweet solution (Stouffer et al., 2015). Insulin is released from pancreatic beta cells in response to increases in circulating blood glucose following the consumption of a meal (Banks et al., 2012). Insulin receptors exist in multiple brain regions, but little is known about how quickly endogenous insulin surges reach and act upon these sites in the central nervous system (Schulingkamp et al., 2000). The ventral striatum, a brain region in the mesolimbic 'reward' pathway, is abundant with insulin receptors. Therefore it is important to investigate insulin's possible role as a signal that communicates nutritive value of recently consumed foods.

Both the function of the mesolimbic pathway and insulin action are highly susceptible to changes in diet. For example, food-restricted rats typically display low circulating insulin levels, lowered basal levels of extracellular dopamine, lowered reward thresholds, and enhanced preferences for cues that predict nutritive food; while diet-induced-obese rats with high circulating insulin exhibit changes associated with a blunted reward response (Ackroff \& Sclafani, 2015; Carr, 2011; Fedorchak \& Bolles, 1987; Geiger et al., 2009). Most recently, it was shown that direct application of insulin to striatal slices increases evoked dopamine release, with an enhanced effect in slices obtained from food-restricted rats and a blunted response in slices from diet-induced obese rats (Stouffer et al., 2015). These diet models highlight a relationship between insulin and striatal dopamine that may have implications for adaptive and maladaptive ingestive behavior. 
Sweet taste or the ingestion of sugar, studied separately using sham-feeding or intragastric (IG) infusions, both increase dopamine release in the striatum (de Araujo et al., 2012; Fernstrom et al., 2012; Tellez et al., 2013). Pairing oral consumption or IG infusion of sugar with a conditioned stimulus (CS), such as an arbitrary flavor, can condition a preference for that CS over a non-paired CS as seen in 'flavor-flavor conditioning' or 'flavor-nutrient conditioning,' respectively. Dopaminergic activity in the NAc is necessary for the formation of both flavor-flavor and flavor-nutrient preferences (Bernal et al., 2008; Touzani et al., 2008). Insulin is released in response to food consumption and has the ability to alter striatal dopamine activity (McCaleb \& Myers, 1979; Owens et al., 2005; Stouffer et al., 2015; Williams et al., 2007; Zhen et al., 2006); such findings led us to investigate insulin's role in the NAc during flavor-nutrient conditioning.

With the ready availability of high caloric foods, and the associated prevalence of obesity, type II diabetes, and eating disorders in Western societies, understanding insulin effects in the brain may be critically important in illuminating underlying mechanisms and have implications to these major public health threats. Given insulin's ability to pass the blood brain barrier after the consumption of a meal and act on the mesolimbic pathway, we tested the hypothesis that insulin serves as a reward signal, reflecting the glycemic load of a recently ingested meal. In a series of experiments, we investigated how consumption of glucose under normal conditions and in various diet models associated with altered peripheral insulin signaling may alter NAc insulin receptor activation. We also examined whether diet-induced changes in insulin or pharmacological manipulations to NAc insulin signaling modify learned food preferences.

\section{Experiment 1a-c: NAc Insulin Receptor Activation Post Ingestion of a Glucose Solution}

In our first series of experiments we explored insulin receptor activation in the NAc after ingestion of a glucose solution. In experiment 1a, we compared NAc tissue samples from rats that received IG infusions of $8 \mathrm{ml}$ water or $16 \%$ glucose. Samples were collected at 7, 17 and 27 minutes after the start of IG infusion to evaluate how quickly glucose infusion can alter activity of the insulin receptor and the downstream signaling protein, Akt, in the NAc. Because insulin is known to enhance striatal dopamine release, we also evaluated the level of phosphorylated AMPA receptor GluA1 at Ser845, a phosphorylation site associated with protein kinase A-mediated signaling downstream of the D1 dopamine receptor and associated with reward and synaptic plasticity (Banke et al., 2000; Carr et al., 2010; Roche et al., 1996; Snyder et al., 2000; Stouffer et al., 2015).

In experiment 1b, we aimed to confirm our findings from experiment 1a in which IG glucose rapidly stimulated insulin receptor activation in the NAc. We allowed rats to voluntarily consume $8 \mathrm{ml}$ of $16 \%$ glucose or water for 7 minutes in their home cage. We then measured levels of insulin receptor, Akt and GluA1 phosphorylation in the NAc.

Experiment $1 \mathrm{a}$ and $1 \mathrm{~b}$ indicated that insulin receptor activation is enhanced at 7 minutes after the onset of glucose infusion or ingestion (see results). Experiment 1c aimed to investigate how these findings may vary in different diet models with altered blood insulin levels. Rats with low blood insulin levels show increased transport of insulin across the blood brain barrier (W. A. Banks et al., 1997). Conversely, evidence exists that diet-induced obese animals display lower levels of insulin in brain tissue and have decreased transport of insulin across the blood brain barrier (Banks et al., 2012; Baskin et al., 1985; Kaiyala et al., 2000). Therefore, in experiment 1c, we explored NAc insulin receptor activation after IG infusions of water or glucose in three different diet models - ad libitum fed $(A L)$, food restricted $(F R)$ and diet-induced-obese $(O B)$ rats. All NAc tissue samples were collected at 7 minutes after the onset of the IG infusions.

\section{Methods for Experiment 1a-c}

\subsection{Rats}

The rats were adult male Sprague-Dawley rats born in our lab or purchased from Taconic Farms (Germantown, NY) or Charles River (Wilmington, MA) weighing 345-423 g (10-12 weeks old) 
at the start of the experiments. They were maintained in a central animal facility with a 12-h light/ dark period (lights on at $6 \mathrm{am}$ ). The rats were singly housed in plastic cages with bedding and free access to standard lab pellets (Rodent Diet \#5001, Lab Diet, St. Louis, MO) and water, except at least one hour before and during the time spent in IG infusion chambers, behavioral conditioning and testing sessions or after assigned a diet regime.

Diet Regimes: In experiment 1c, rats were randomly assigned to three diet groups: food restricted (FR), food ad libitum (AL), and diet-induced obesity (OB). Post recovery from IG surgery, rats in the FR group were given $10 \mathrm{~g}$ chow/day, representing $\sim 40 \%$ of ad libitum intake, until they reached $80 \%$ of their surgical recovery weight. Food was then titrated daily to maintain the $80 \%$ weight level. The FR rats were maintained on this regime for 24-25 days prior to test day.

Rats in the OB group were given unlimited access to Milk Chocolate Ensure (Abbott Nutrition,Columbus, $\mathrm{OH}$ ) in addition to standard rat chow, beginning about 10 days before IG surgery. Unlimited access to Ensure continued throughout the remainder of the study and was removed only during transport to, and during IG infusion sessions. Rats in the OB group had 45-46 days of unlimited access to Ensure prior to test day.

In experiment $1 \mathrm{a}$, each group consisted of the following number of rats: 7 minute IG glucose $\mathrm{n}$ $=5,17$ minute IG glucose $n=5,27$ minute IG glucose $n=5,7$ minute IG water $n=4,17$ minute IG water $n=5,27$ minute IG water $n=5$. In experiment $1 b$, each group consisted of the following number of rats: oral glucose $n=8$, oral water $n=6$. In experiment $1 \mathrm{c}$, the number of rats per group was as follows: $A L$ IG water $n=4, A L$ IG glucose $n=5, F R$ IG water $n=5, F R$ IG glucose $n=6$, OB IG water $n=5, O B$ IG glucose $n=6$.

Experimental procedures were approved by the Institutional Animal Care and Use Committee at the New York University School of Medicine and were consistent with the Principles of Laboratory Care (NIH Publication no. 85-23).

\subsection{Surgery}

Intragastric surgery: Rats were fitted with IG catheters (size: $3 F r(0.6 \mathrm{~mm} I D \times 1.0 \mathrm{~mm}$ OD) Polyurethane tubing, C30PU-RJV1303, Instech Laboratories, Plymouth Meeting, PA) while anesthetized with ketamine (100 mg/kg, i.p.) and xylazine $(10 \mathrm{mg} / \mathrm{kg}$, i.p.). Rats were given 30,000 units of penicillin (IM) at the time of surgery. The catheter entered the fundus and was secured with Dacron mesh (DCMESH, Instech Laboratories). The catheter passed subcutaneously from the peritoneum to between the shoulders, where it was attached to a Luer lock Vascular Access Button (VAB95BS, InsTech Laboratories). Postsurgical analgesia was achieved by twice daily administration of Banamine (flunixin, $1.1 \mathrm{mg} / \mathrm{kg}$, s.c.) for three days. Catheters were flushed with $0.2 \mathrm{ml}$ water daily. Rats were weighed regularly and given a minimum of three weeks to recover before habituation procedures began.

\subsection{Apparatus}

For IG experiments (1a and 1c), habituation and testing was conducted in infusion cages. In the infusion cages, the Luer lock Vascular Access Button (the entry point for the rat's catheter) was attached to a Luer stub/Tether (KVAB95T, Instech Laboratories) which connected the catheter with polyurethane tubing that passed through an infusion swivel mounted on a counterbalanced lever. The tubing was connected to a syringe mounted on a pump set at a $1.3 \mathrm{ml} / \mathrm{min}$ infusion rate.

\subsection{Solutions}

During habituation sessions that included an IG infusion, all rats received an $8 \mathrm{ml}$ IG water infusion. On test day, rats received either an $8 \mathrm{ml} \mathrm{IG}$ infusion of water or $16 \%$ glucose (G8270, Sigma-Aldrich, St. Louis, MO). This infusion volume is within the range used in IG conditioning studies (Ackroff et al., 2010; Yiin et al., 2005a).

\subsection{Procedure}

Intragastric Infusions Sessions: For all sessions, rats had no access to food and water when transported from the central animal facility to the procedure room and in the room. They were weighed and were left undisturbed for an hour to habituate to the procedure room. The rats received 
six habituation sessions, each lasting 27 minutes. During the first two sessions, they were attached to the infusion tether but no solution was infused. During the next four habituation sessions $8 \mathrm{ml}$ of water was infused at the beginning of the session. On the test day, the rats were infused with water or $16 \%$ glucose and sacrificed at three different time points after the onset of the infusion. In experiment 1a, assignment into the four groups (7-27 minute water, 7 minute glucose, 17 minute glucose and 27 minute glucose) was semi-random, counterbalancing for weight and ensuring that each group included rats tested earlier and later in the day. In experiment 1c, all rats were sacrificed at $7 \mathrm{~min}$ post-infusion and were semi-randomly assigned to receive glucose or water within diet treatment, and counterbalanced for weight and time of day.

Drinking Sessions: All sessions occurred in the rat's home cage. Rats were semi-randomly assigned, ensuring equal weights, into two groups: water or $16 \%$ glucose. The ad libitum fed rats were given two training sessions with access to their assigned solution in order to reduce neophobia to the glucose solution and encourage them to consume the glucose within 7 minutes. Six days after the last training session, rats were given 7 minutes to drink $8 \mathrm{ml}$ of water or $16 \%$ glucose before being sacrificed for tissue collection as described in experiment 1a. Fluid intake was measured by weighing the bottles before and after the session.

ELISA: Trunk blood was collected in BD Vacutainer blood collection tubes $(367989$, Becton Dickinson, Franklin Lakes, NJ) from each rat at the time of sacrifice. Blood collection tubes were left upright for 30 minutes post collection and then centrifuged at $3000 \times \mathrm{g}$ for $15 \mathrm{~min}$ at $25^{\circ} \mathrm{C}$. Serum was collected, aliquoted and stored at $-80^{\circ} \mathrm{C}$. Levels of insulin were determined using an Insulin ELISA kit according to the manufacturer's protocol (80-INSHU-E01, ALPCO Diagnostics, Salem, NH).

Whole-Cell Preparation: Rats were briefly exposed to $\mathrm{CO} 2$ and decapitated by guillotine. Brief CO2 exposure prior to decapitation or cervical dislocation is common in rodent brain biochemical studies and has been found not to alter brain regional levels of cholinergic markers or biogenic amine neurotransmitters (Jones et al., 1999). Effects of this humane method of euthanasia on protein phosphorylation have not been systematically examined, though the method is applied consistently across subjects and treatment groups. Brains were extracted and rapidly frozen in powdered dry ice. A series of 500- $\mu \mathrm{m}$ sections were cut using an IEC Minotome cryostat, and NAc was dissected using a combination of micropunch and microknife under an Olympus dissecting microscope. All western data examined differences in the NAc, encompassing both the NAc core and NAc shell. Tissue samples were then homogenized in Lysis Reagent (C3228, Sigma-Aldrich, St. Louis, MO) and manufacturer recommended concentrations of Phosphatase Inhibitor Cocktails I and II and Mammalian Protease Inhibitor Cocktail (P5726; P0044; 11873580001, all from Sigma-Aldrich, St. Louis, MO). The homogenates were centrifuged at $30,000 \times \mathrm{g}$ for $10 \mathrm{~min}$ at $4^{\circ} \mathrm{C}$, and the nonsolubilized fraction was discarded. Lysates were then mixed with 4x Laemmli Sample Buffer (1610747, Bio-Rad Laboratories, Hercules, CA) mixed with $\beta$-mercaptoethanol and warmed at $37^{\circ} \mathrm{C}$ degrees for 30 minutes. Unused denatured lysate was stored in sample buffer in aliquots at $-80^{\circ} \mathrm{C}$. The protein content was determined by the BCA Bradford Assay (23223, Thermo Scientific, Rockford IL) using bovine serum albumin as standard.

Western Blotting: Western blotting Proteins (10-25 $\mathrm{gg} / \mathrm{lane})$ were separated by electrophoresis on precast 4-12\% sodium dodecyl sulfate polyacrylamide gels (Lonza, Rockland, ME). Precision Plus Protein standard molecular weight markers (Bio-Rad) were loaded to estimate the size of the target proteins and to ensure complete transfer of proteins from gel to membrane. Proteins were electrophoretically transferred to Protran nitrocellulose membranes (Whatman, Mobile, $A L$ ) for $1.75 \mathrm{~h}$ at a constant voltage of $100 \mathrm{~V}$. Membranes were blocked for $60 \mathrm{~min}$ with $5 \%$ BSA TBS (BP1600, Fisher BioReagents, Pittsburgh, PA) with shaking at room temperature. Membranes were then probed overnight at $4{ }^{\circ} \mathrm{C}$ using primary antibodies for target proteins or the protein loading control, $\alpha$ tubulin. Primary antibodies were diluted in 5\% BSA TBST with $0.2 \%$ Tween 20 . After probing with primary antibodies and washing with TBST buffer $(3 \times 5 \mathrm{~min})$, membranes were incubated for $1 \mathrm{~h}$ at room temperature with IRDye 680RD Goat anti-Mouse IgG (H+L) and IRDye 800CW Goat anti-Rabbit 
$\lg (\mathrm{H}+\mathrm{L})(1: 16,000$; LI-COR Biosciences) in 5\% BSA TBST with $0.2 \%$ Tween 20. Proteins were visualized using an Odyssey CLx infrared imaging system, and bands were quantified using the Image Studio software (LI-COR Biosciences). Samples from each of the four test groups were loaded in each gel in an alternating order. The pattern of alternation differed between the multiple gels run to obtain averaged results for each target protein in each test group.

Antibodies: Primary antibodies used included rabbit polyclonal antiphospho-Tyr1162/1163-IRß (1:200; sc-25103, Santa Cruz Biotech, Santa Cruz, CA), mouse monoclonal anti-IRß (1:1500; 3020, Cell Signaling, Danvers, MA),rabbit monoclonal antiphospho-Ser473-Akt (1:1000; 4060, Cell Signaling, Danvers, MA), mouse monoclonal anti-Akt (1:1000; 4685, Cell Signaling, Danvers, MA), mouse monoclonal anti-GluA1 (1:1000; MAB2263, Millipore, Temecula, CA), rabbit polyclonal antiphospho-Ser845-GluA1 (1:1000; AB5849, Millipore), and mouse monoclonal anti-a-tubulin (1:10,000; T6199, Sigma-Aldrich, St Louis, MO).

\subsection{Verification of Catheter Placement}

After rats were briefly exposed to $\mathrm{CO} 2$ and decapitated by guillotine, we confirmed placement of the IG catheter. Blue dye was infused into the cathether and if the inside of stomach turned blue with no leaks, the catheter placement was confirmed. All catheters were confirmed in place at the end of each study.

\subsection{Analysis}

For experiments 1a-c, we predicted that IG infusion or oral consumption of glucose would induce insulin release, leading to enhanced striatal insulin activation. Therefore, individual independent one-tailed t-tests were performed between rats that received water and glucose. No additional corrections were made for the number of comparisons. In experiment 1a, the water group included rats sacrificed at 7, 17, and 27 minutes post intragastric infusion of water. In experiment 1c, the effects of IG solution (water or glucose) and diet (FR, AL, or OB) on phosphorylation of the insulin receptor was analyzed in a 2 x 3 ANOVA. Given our prior finding (Stouffer et al., 2015) that diet impacts striatal insulin response, follow-up pairwise comparisons were made between IG solutions within each diet group. Analysis was conducted using SPSS and Excel Data Analysis tool pack.

\section{Results}

\subsection{Experiment 1a:}

There was a significant increase in the level of phosphorylated/total IR Tyr1162/1163 in rats that were sacrificed 7 minutes after IG $16 \%$ glucose compared to rats that received IG water infusion $(t(7)=2.10, p<0.05)$ (Fig 1a). Thus a glucose solution is able to stimulate insulin receptor activation in the NAc rapidly. There were no significant differences between water and the 17 minute glucose group $(t(8)=0.67, p=0.26)$ or 27 minute glucose group $(t(8)=0.15, p=0.44)$.

Investigation of Akt, a downstream component in the insulin signaling pathway, also showed rapid activation. Analysis revealed a significant increase in the level of phosphorylated/total Akt Ser473 in rats that were sacrificed 7 minutes post glucose infusion compared to rats that received an IG water infusion $(t(7)=2.57, p<0.025)$ (Fig 1b). However, there were no significant differences in phosphorylated/total Akt Ser473 between the water and 17 minute $(t(8)=0.41, p=0.35)$ or 27 minute glucose group $(t(8)=0.11, p=0.46)$.

Given the previous finding (Stouffer et al., 2015) that insulin increases evoked dopamine release, we also probed phosphorylation of GluA1 at Ser845, which is increased in response to dopamine receptor 1 activity. Rats sacrificed 7 minutes post glucose infusion had significantly higher levels of phosphorylated/total GluA1 Ser845 compared to the water infused rats $(t(7)=2.37, p<0.05)$ (Fig 1c). Rats sacrificed at 17 and 27 minutes post glucose infusion did not show a significant increase in phosphorylated/total GluA1 Ser845 $(t(8)=0.02, p=0.49)(t(8)=0.57, p=0.29)$. Further investigation will be required to confirm direct dependence of Akt phosphorylation on insulin signaling and GluA1 phosphorylation on dopamine signaling. 
(a) Nucleus Accumbens Insulin Receptor
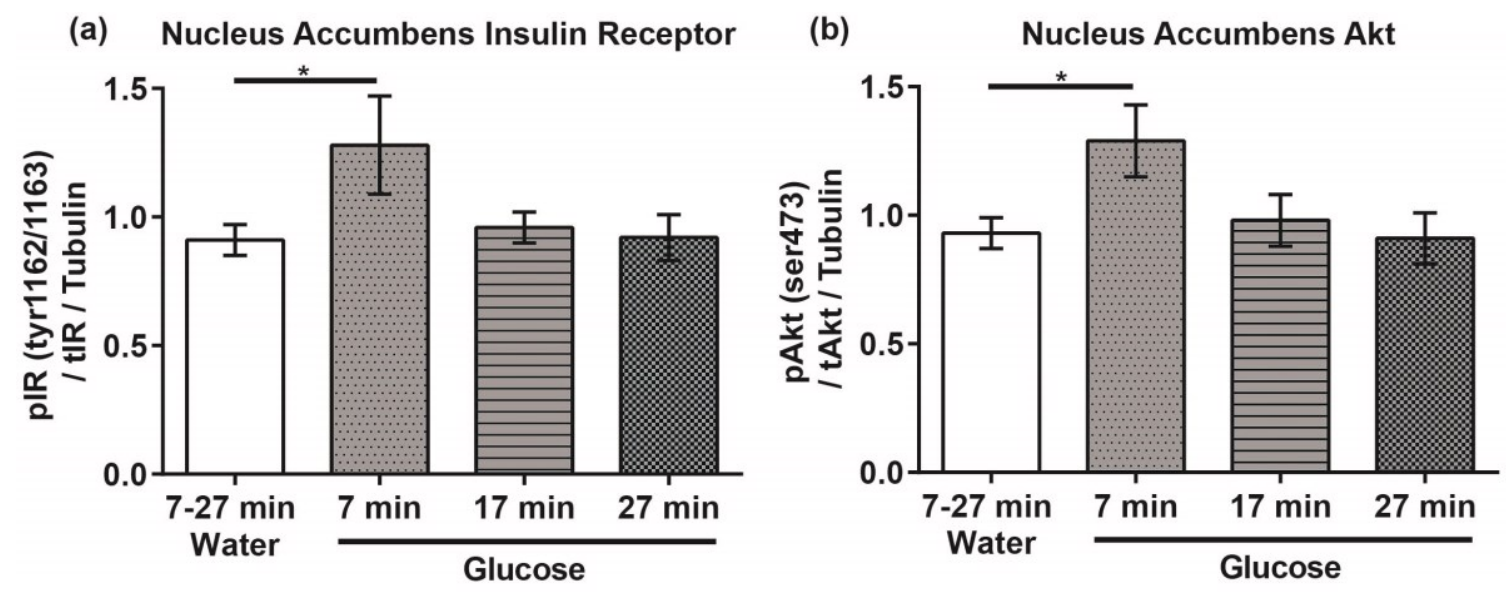

Time Post Onset of Intragastric Infusion

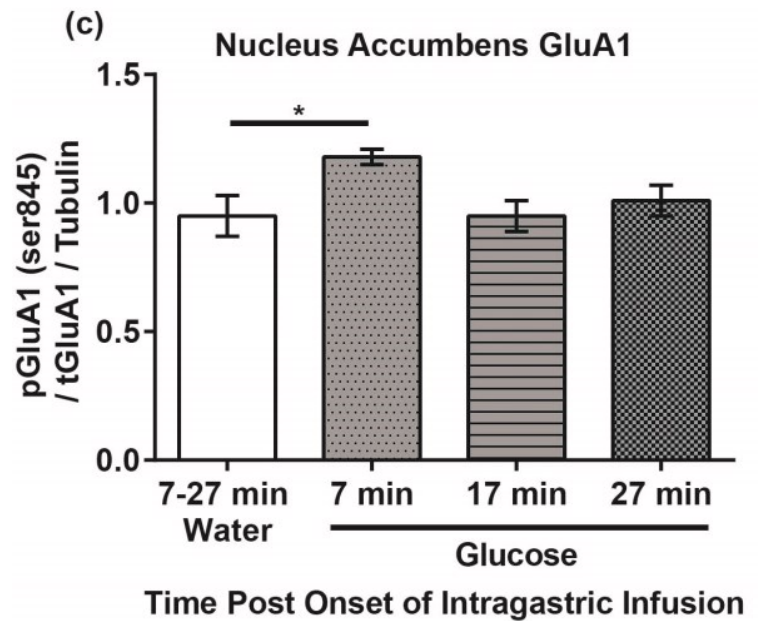

(d)

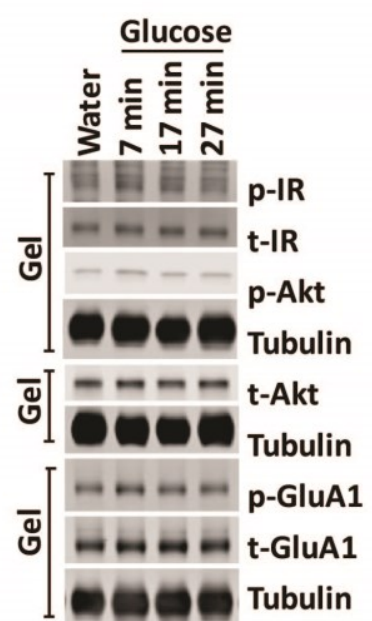

Figure 1 Insulin activity in the NAc rapidly increases post intragastric infusions of a $16 \%$ glucose solution. (a) Phosphorylation of the insulin receptor at Tyr1162/1163, (b) Phosphorylation of the Akt at Ser473, and (c) Phosphorylation of GluA1 at Ser845 7, 17 and 27 minutes post the onset of an infusion of water or glucose. (d) Representative blots.

\subsection{Experiment 1b:}

During the 7 minute drinking session, rats given 16\% glucose drank significantly more fluid than rats given water ( 8 vs. $4 \mathrm{ml}, \mathrm{t}(12)=7.92, \mathrm{p}<0.0005)$. Oral glucose consumption significantly increased blood insulin levels above that of the water group $(t(12)=1.78, p<0.01)($ Fig $2 e)$. Consistent with findings from experiment $1 \mathrm{a}$, rats that consumed glucose showed increases in phosphorylated/total IR Tyr1162/1163 $(\mathrm{t}(12)=1.99, \mathrm{p}<0.05)$, phosphorylated/total Akt Ser473 $(\mathrm{t}(12)=2.11, \mathrm{p}<0.05)$, and phosphorylated/total GluA1 Ser845 compared to the water control group $(\mathrm{t}(12)=2.26, \mathrm{p}<0.025)($ Fig 2a-d). 

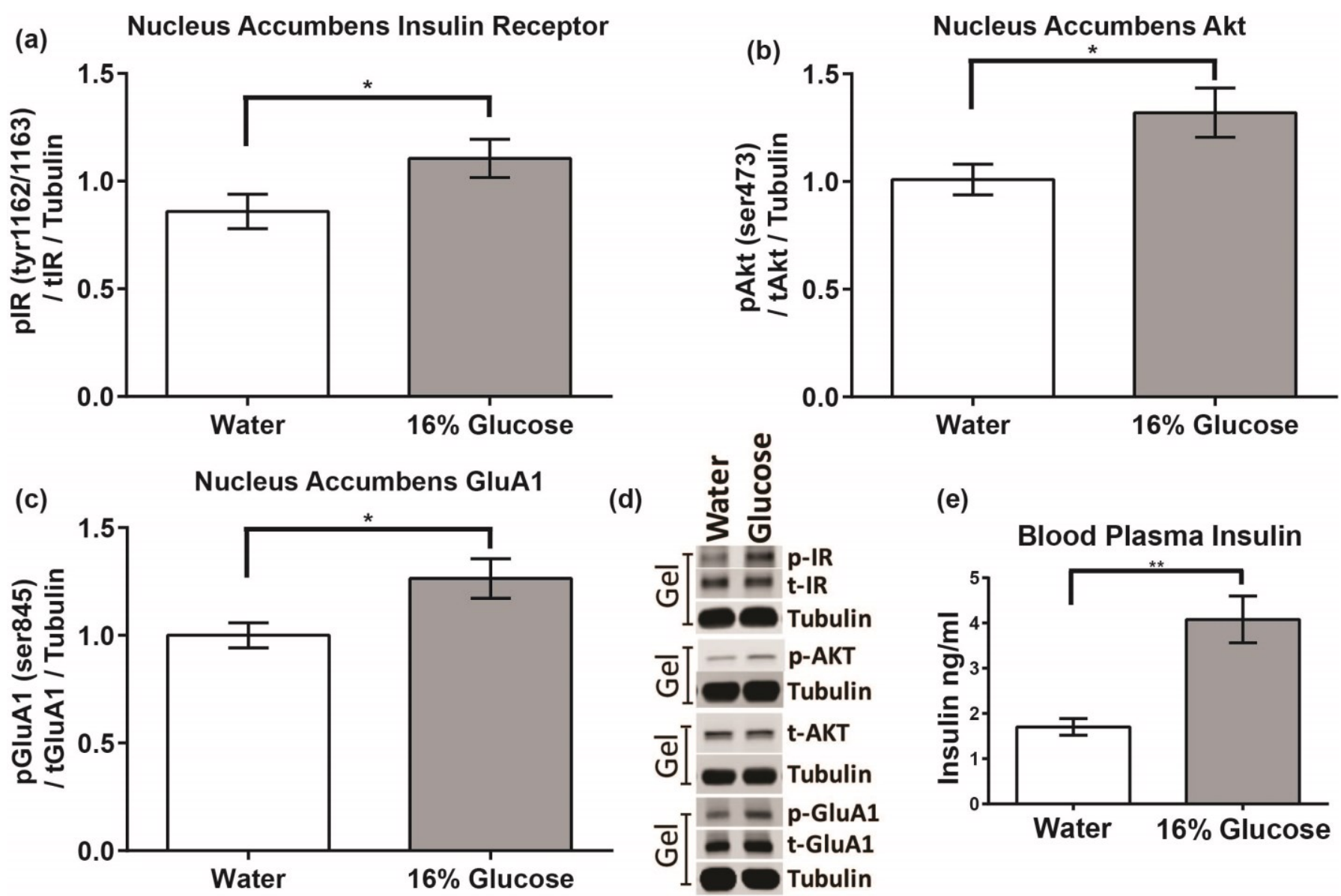

(e)

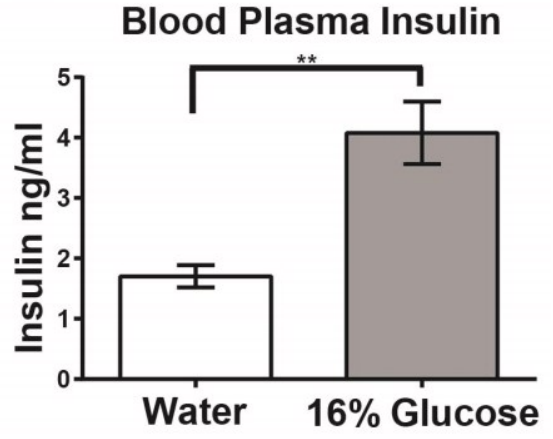

Figure 2 Peripheral increases in insulin secretion induced by oral consumption of a glucose solution are accompanied by increases in NAc insulin activity. (a) Phosphorylation of the insulin receptor at Tyr1162/1163, (b) Phosphorylation of Akt at Ser473, (c) Phosphorylation of GluA1 at Ser845, and (e) Blood plasma insulin levels ( $\mathrm{ng} / \mathrm{ml}$ ) after 7 minute access to a $16 \%$ glucose solution or water. (d) Representative western blots.

\subsection{Experiments 1c}

Diet had a significant impact on body weight $\left(F_{2,28}=372.99, p<0.001\right)$; all diet groups were significantly different from one another $(F R<A L<O B)$ (Fig 3a) $(A L$ vs $O B t(19)=13.54, p=7.76 E-11$; $A L$ vs FR t(19)=14.79, $p=3.06 \mathrm{E}-10 ; \mathrm{FR}$ vs OB $\mathrm{t}(20)=25.47, \mathrm{p}=8.82 \mathrm{E}-13)$. Both IG solution $\left(\mathrm{F}_{1,25}=9.86\right.$, $p<0.01)$ and diet $\left(F_{2,25}=8.03, p<0.01\right)$ had a significant impact on insulin receptor phosphorylation. However, there was no significant interaction between diet and IG solution $\left(F_{2,25}=0.67, p=0.52\right)$. Follow up comparisons, justified by the a priori prediction of diet group differences based on our previous study (Stouffer et al., 2015) revealed significant differences in insulin receptor phosphorylation between IG water and glucose in the $A L$ rats $(t(7)=1.95, p<0.05)$ and FR rats $(t(9)$ $=3.75 p<0.01)$, but not OB rats $(t(9)=1.10, p=0.15)($ Fig $3 b-c)$. 

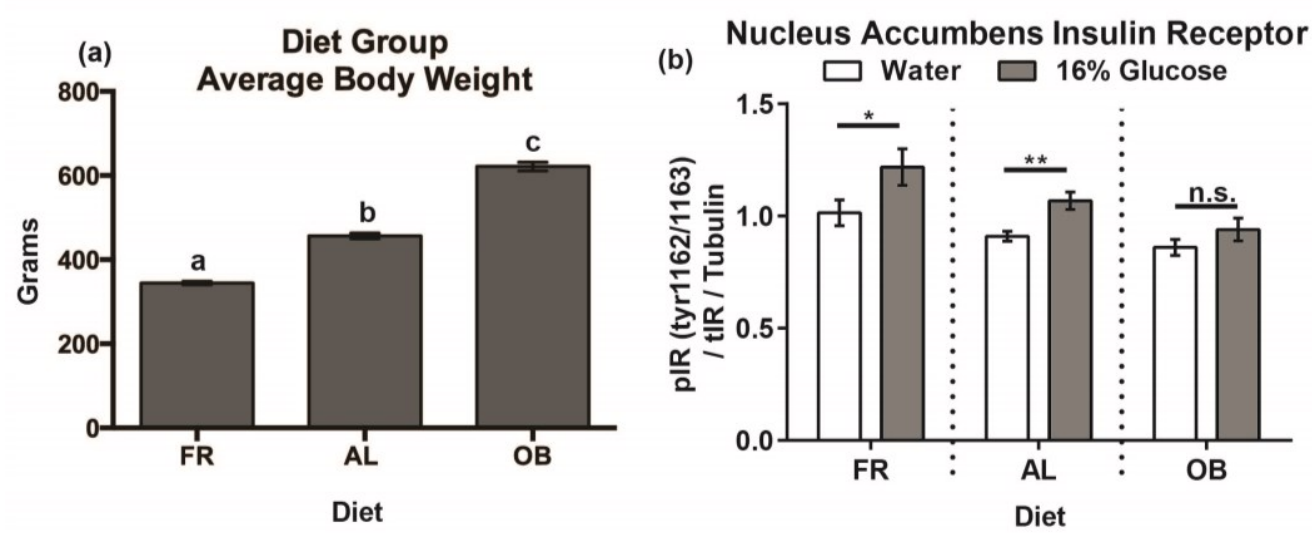

(c)

Figure 3 Diet impacts NAc insulin activity. (a) Average body weight per diet group at the time of sacrifice. Letters (a,b,c) indicate a significant difference from each group. (b) Phosphorylation of NAc insulin receptor at Tyr1162/1163 after a 7-minute intragastric infusion of $16 \%$ glucose. (c) Representative blots. (AL: ad libitum, OB: diet-induced obesity, FR: food restricted).

\section{Experiment 2: The role of NAc Insulin Receptor Activity in Flavor-Nutrient Preference}

In our first series of experiments, we established that consumption of a glucose solution stimulates an increase in insulin receptor activation in the NAc. Previous research from our lab also demonstrates that insulin may contribute to processing the rewarding value of a recently consumed meal (Stouffer et al., 2015). However, our previous results do not clarify insulin's role in communicating the nutrient value of a recently consumed meal. Therefore, in the second series of experiments, we explored insulin's role in signaling the nutritive value of a sweet solution.

In experiment 2a, we employed a flavor-nutrient conditioning paradigm modified from Warwick and Weingarten (Warwick \& Weingarten, 1994). Their study determined that a $6.1 \%$ glucose (G) solution and a $1 \%$ glucose $+0.125 \%$ saccharin $(\mathrm{g}+\mathrm{s})$ solution were equally preferred by sham-feeding rats. Naïve rats were then conditioned to associate a flavor (the CS+, e.g. cherry) mixed in the high glucose solution (G) and a different flavor (the CS-, e.g., grape) mixed in the low glucose solution $(\mathrm{g}+\mathrm{s})$ during separate 1-bottle $24-\mathrm{h}$ training sessions. After 16 training sessions, the rats were given a two-bottle choice test in which both CS flavors were presented in water. The rats demonstrated a preference for the CS+ flavor previously paired with $\mathrm{G}$. Because the solutions were equally palatable to sham-fed rats, the preference for the CS+ flavor demonstrated the rats' ability to sense the nutritive differences between the two solutions and assign higher value to the CS+ flavor. In order to use the Warwick and Weingarten protocol with brief pharmacological manipulations (described in experiment 2c), we investigated if rats can develop a flavor-nutrient preference with fewer conditioning and shorter conditioning sessions.

Experiment 1c showed that glucose-stimulated NAc insulin receptor activation was greatest in FR rats and somewhat less in AL rats; however, OB rats showed little change. If NAc insulin activity contributes to sensing and learning about the glycemic consequences of recently consumed solutions, then we predict FR rats to be hypersensitive to changes in insulin and show an enhanced flavor-nutrient preference. OB rats may show a decreased response to insulin and fail to acquire a flavor-nutrient preference. Therefore in experiment $2 a$, we conditioned FR, AL and OB rats in our modified flavor-nutrient preference paradigm.

In experiment $2 \mathrm{~b}$, we investigated whether NAc insulin receptor activation reflects glycemic yield of a glucose solution rather than just the absence or presence of glucose. We used conditioned rats from experiment $2 a$ to determine if there are differences in NAc insulin receptor phosphorylation in rats consuming equal amounts of the flavored $\mathrm{G}$ or $\mathrm{g}+\mathrm{s}$ solutions.

In experiment 2c, we used a pharmacological manipulation to further investigate insulin's role in the NAc in flavor-nutrient conditioning. We hypothesized that inhibiting NAc insulin activity during conditioning sessions with $\mathrm{G}$ solution would impair the ability to detect the signal derived from that solution and thus impair the ability to acquire a flavor-nutrient preference. Infusions of insulin antibody 
have often been used to investigate insulin's role in ingestive behavior and cognition (McNay et al., 2010; Paranjape et al., 2010; Stouffer et al., 2015; Strubbe \& Mein, 1977). Moreover, the specific antibody used here was previously verified to inactivate insulin and prevent its effect on DA transport in striatal synaptosomes (Stouffer et al., 2015).

In addition to findings from Stouffer et al 2015, the present experiment $2 \mathrm{~d}$ tested the ability of InsAb to prevent insulin receptor phosphorylation in response to glucose consumption. Experiment $1 \mathrm{~b}$, comparing NAc tissue after 7 minute access to water or $16 \%$ glucose, was repeated in FR rats. After confirming that oral consumption of $16 \%$ glucose increased NAc IR activation, we compared NAc samples from FR rats that received microinjections of IgG or InsAb prior to 7 minute access to glucose.

\section{Methods}

\subsection{Rats}

Rats and diet protocols used in experiments 2a-c were similar to those described in experiment 1. Food was removed from home cages during all behavioral sessions, except overnight testing (see below). In experiment $2 a$, baseline testing occurred before diet regimes began for all three diet groups: AL, FR and OB. After 4 weeks of diet treatment, all rats began conditioning sessions. After flavor-nutrient testing for $\mathrm{AL}$, FR and OB groups was complete, an additional FR group (FR - limited intake) was conditioned with limited access to conditioning solutions to determine whether the elevated intakes during conditioning influenced the preference of the FR group.

The following number of rats were used per group in experiment $2 a: A L n=20, F R / F R-$ limited intake $n=23(12 / 11), O B=12$. In experiment $2 b, A L n=11$. In experiment $2 c, \lg \mathrm{n}=8$, InsAb $n=9$. In experiment $2 d$, FR water $n=5$, FR glucose $n=5$, FR glucose IgG $n=5$, FR glucose InsAb $n=6$. Rats receiving NAc shell microinjections in experiment $2 \mathrm{~d}$ were used in an unrelated study prior to this study, in which they received 4 microinjections of saline.

\subsection{Surgery}

In experiment $2 c$ and $2 d$, rats were anesthetized with ketamine (100 mg/kg, i.p.) and xylazine (10 mg/kg, i.p.). They were stereotaxically implanted with chronically indwelling guide cannulae. Two guide cannulae (26 ga) were placed bilaterally $2.0 \mathrm{~mm}$ dorsal to the injection sites in the NAc shell (1.6 $\mathrm{mm}$ anterior to bregma; $2.1 \mathrm{~mm}$ lateral to the sagittal suture, tips angled $8^{\circ}$ toward the midline, $5.8 \mathrm{~mm}$ ventral to skull surface). Cannula patency was maintained with occlusion stylets. The cannulae and four mounting screws were then permanently secured to the skull by flowing dental acrylic around them. Postsurgical analgesia was achieved by administration of Banamine (flunixin, $2.0 \mathrm{mg} / \mathrm{kg}$, s.c.).

\subsection{Apparatus}

All conditioning and testing in experiments $2 \mathrm{a}$ and $2 \mathrm{~b}$ occurred in rats' home cages in the central animal facility. For all behavioral procedures in experiment 2c (except specified test sessions, see below), rats were weighed in the central animal facility before being transported in home cages to a procedure room. Once there, all rats remained undisturbed for $30 \mathrm{~min}$ before food hoppers were removed and bottle holders placed in cages, which were used to present bottles during conditioning and testing sessions. Fluid intake was measured by weighing the bottles before and after the sessions.

\subsection{Solutions and Drugs}

In experiments 2a-2c, there were two sweet solutions: a $6.1 \%$ glucose (G, Sigma-Aldrich) solution and a $1 \%$ glucose plus $0.125 \%$ sodium saccharin ( $g+s$, S1002,Sigma-Aldrich) solution. During conditioning, cherry or grape $(0.05 \%$ Kool-Aid powder, Kraft Foods, Northfield, IL) was added to the sweet solutions. The CS+ flavor was added to the $6.1 \%$ glucose solution $(\mathrm{G})$, while the CSflavor was added to the $1 \%$ glucose plus $0.125 \%$ saccharin solution $(\mathrm{g}+\mathrm{s})$. Flavor-solution pairs were counterbalanced so that half of the rats received grape as the CS+ and cherry as the CS-; the flavors 
were reversed for the remaining rats. Experiment $2 \mathrm{~d}$ used the same solutions as experiment $1 \mathrm{a}-\mathrm{c}$, water and $16 \%$ glucose (G8270, Sigma-Aldrich, St. Louis, MO).

The intracranial infusion solutions used in experiment $2 \mathrm{c}$ and $2 \mathrm{~d}$ were insulin antibody (InsAb; $0.5 \mu \mathrm{l}$, undiluted, $1.0 \mathrm{mg} / \mathrm{ml}$, Abcam ab46707, Cambridge, MA) and immunoglobulin G (lgG; $0.5 \mu \mathrm{l}$, $1.0 \mathrm{mg} / \mathrm{ml}$, Abcam ab81032, Cambridge, MA). IgG is an antibody targeting a synthetic protein that does not exist in the rat brain; its use as a control serves to demonstrate that infusions of an antibody alone are not aversive. For microinfusions, two $30 \mathrm{~cm}$ lengths of PE-50 tubing were attached on one side to $25-\mu \mathrm{l}$ Hamilton syringes filled with distilled water, and on the other side to 31-guage injector cannulae extending $2.0 \mathrm{~mm}$ beyond the end of the implanted cannula guides. Rats were placed on a cushion and their stylets were removed. Injectors were inserted and $0.5 \mu$ infusions were made over a period of $90 \mathrm{sec}$. Injector cannulae were left in place for another minute before being removed, at which point stylets were replaced and the rat returned to its home cages for behavioral testing. Mock infusions were identical to antibody infusions, except that no solution was infused and injector needles were shorter to prevent piercing brain tissue.

\subsection{Procedure}

Habituation: Habituation sessions only occurred in experiment 2c. During the first five sessions, rats were habituated to handling and transport from the central animal facility to the procedure room. In order to mimic the infusion and behavioral procedure, rats were held on a cushion for 3 minutes and their stylets were removed and replaced. After 3 minutes, rats returned to their home cage with food hoppers removed and bottle holders in place (no bottles) for a period of $30 \mathrm{~min}$. After 30 minutes, bottle holders were removed and food hoppers were replaced.

Baseline consumption of unflavored solutions: To verify that naive rats do not prefer the higher glucose solution prior to conditioning, we performed a baseline preference test without flavors. All rats were given 30-min access to the unflavored $\mathrm{G}$ and g+s solutions. To control for side bias, the left-right position of the $G$ and $g+s$ solutions were counterbalanced across rats and alternated over two days.

Flavor-nutrient preference conditioning: During flavor-nutrient conditioning, the rats were given one-bottle, 30-minute access to $\mathrm{CS}+/ \mathrm{G}$ and $\mathrm{CS}-\mathrm{g}+\mathrm{g}$ solutions on alternate days. Half the rats began with a CS+ conditioning session, while the other half began with a CS- conditioning session. There was a minimum of 24 hours between each conditioning session. In experiments $2 a$ and $2 b$, without pharmacological manipulations, there were a total of 8 conditioning sessions. In experiment $2 a$, the solution in the bottles given to FR-limited rats was limited to the average intake of AL rats in each conditioning session.

In experiment 2c, ad libitum fed rats were semi-randomly assigned to receive one of the two antibody microinfusions (InsAb or IgG) with groups matched for body weight and baseline consumption. Each conditioning session consisted of two parts: a microinfusion of the assigned antibody followed by a conditioning session as described above. On each conditioning session, rats received the assigned antibody prior to a CS+ conditioning session and a mock infusion prior to a CSconditioning session. Each pair of conditioning sessions (antibody and mock) was counterbalanced across rats within antibody treatment. All rats underwent $10 \mathrm{CS}+$ conditioning sessions and $10 \mathrm{CS}-$ conditioning sessions, for a total of 20 sessions.

Testing: During the choice test, the rats were presented with two bottles containing the CS+ and CS- flavors in water (without sweeteners). Two test sessions were conducted with the side placements counterbalanced across days to control for any side bias, i.e., one test is data from two consecutive overnight sessions.

Experiment $2 \mathrm{a}$ and $2 \mathrm{~b}$ rats had limited handling and transportation prior to behavioral sessions, and consumed sufficient amounts of CS flavored water in the 30-minute test sessions to demonstrate a flavor preference. However, rats in experiment 2c consumed very little (less than $3 \mathrm{ml}$, data not shown), in the initial 30-minute test session. This difference was likely due to the extra handling and transportation required in the study using intracranial infusions. The 30-minute test session occurred after the $8^{\text {th }}$ conditioning session. To maximize consumption of test solutions in experiment $2 \mathrm{c}$, we 
probed for flavor preference using overnight two-bottle sessions ( $5 \mathrm{pm}$ to $9 \mathrm{am}$ ) in subsequent test sessions. The first four overnight sessions, or 2 tests, occurred after the $10^{\text {th }}$ conditioning session, while the final six overnight sessions, or 3 tests, occurred after all 20 conditioning sessions. All rats had 24-32 hours of inactivity between the last conditioning session and the subsequent test session. Because there were no differences observed during CS- conditioning sessions between InsAb and IgG treated animals, we do not believe InsAb blockade persists beyond a 24 hour period. However, further experimentation is needed to confirm the exact duration of InsAb blockade.

After the final overnight test in experiment 2c, rats were given a 30-minute preference test in the procedure room with their training solutions, i.e., CS+/G vs. CS-/g+s.

Terminal-test sessions: In experiment $2 \mathrm{~b}, \mathrm{AL}$ and FR-limited rats were separated into two groups based on test preference. A week after flavor preference testing, half the rats received their $\mathrm{CS}+/ \mathrm{G}$ solution while the other half received the $\mathrm{CS}-/ \mathrm{g}+\mathrm{s}$ solution. Access to training solutions were limited (12 $\mathrm{ml}$ total) to ensure that rats consumed the same total volume. Based on the rapid increase in NAc insulin receptor phosphorylation observed in experiment 1a, terminal test sessions were limited to 7 minutes. Western blot analysis of tissue samples was performed as described in the procedure section of experiment 1.

Non-conditioning Drinking Session: In experiment 2d, FR rats were given two training sessions with access to their assigned solution (water or $16 \%$ glucose) in order to reduce neophobia to the glucose solution and encourage them to consume the glucose within 7 minutes. Six days after the last training session, rats were given 7 minutes to drink $8 \mathrm{ml}$ of their assigned solution before being sacrificed for tissue collection as described in experiment 1a. Fluid intake was measured by weighing the bottles before and after the session. Half the rats received NAc shell microjinjections of either IgG or InsAb prior to 7 minute access to $16 \%$ glucose. The other half received no microinjections, and were assigned 7 minute access to either water or $16 \%$ glucose.

\subsection{Histology}

After experiment 2c was completed, rats were euthanized with brief exposure to $\mathrm{CO}_{2}$ and decapitated. Brains were removed and placed in 10\% buffered formalin for at least $48 \mathrm{~h}$. The 2800 Frigocut (Cambridge Instrumentals, West Germany) was used to cut $40 \mu \mathrm{m}$ coronal slices. The sections were thaw-mounted on glass slides coated with gelatin and stained with cresyl violet before being cover-slipped with Permount. The placements of the cannulae were ascertained using an Olympus SZ40 microscope. One rat whose cannulae were not placed accurately within the NAc shell was eliminated from data analysis.

\subsection{Analysis}

In experiment $2 a$, average consumption during conditioning sessions was analyzed in a $2 \times 4$ ANOVA, with CS solution as the within subjects factor and diet as the between subjects factor. Follow up two-tailed t-tests examined differences between CS+ and CS- conditioning solution intakes within each diet group. In experiment 2c conditioning sessions were analyzed by a 2x10 mixed ANOVA with repeated measures on conditioning session and microinfusion treatment (InsAb or IgG) as the between subjects factors. Separate ANOVAs were conducted for CS+ and CS-conditioning sessions because microinfusion treatment preceding the sessions was different. Protected one-tailed t-tests for independent samples using pooled error from omnibus ANOVA were also analyzed for each individual conditioning session.

In experiment $2 \mathrm{~b}$, individual one-tailed t-tests comparing NAc insulin receptor phosphorylation were compared between rats receiving CS+ conditioning solution and rats receiving the CSconditioning solution. Comparisons were made within diet group (i.e. AL CS+ vs. AL CS-). Similarly, in experiment $2 \mathrm{~d}$, individual one-tailed t-tests comparing NAc insulin receptor phosphorylation were compared between FR rats received water and 16\% glucose, as well as between FR rats that received IgG and FR rats that received InsAb. No additional corrections were made for the number of comparisons. 
Test preference in experiment 2a was analyzed in a one-way ANOVA with follow up comparisons between diet groups. Individual one-tailed t-tests between the intakes of the CS+ and CS- during testing were conducted to ascertain whether a flavor-nutrient preference was acquired within each diet group. In experiment 2c, test sessions were analyzed by a 2x2 mixed ANOVA with microinfusion treatment (InsAb or IgG) as the between subjects factor. We hypothesized that microinfusions of InsAb, but not IgG, would impair the ability to acquire a flavor preference; therefore, follow up one-tailed t-tests between the CS+ and CS- were performed for both microinfusion groups. Analysis was conducted using SPSS and Excel Data Analysis tool pack.

\section{Results}

\subsection{Experiment 2a Results}

Prior to beginning diet regimes the rats showed a slight but significant preference for the glucose plus saccharin solution $(53 \%, 9.2 \mathrm{vs} .7 .8 \mathrm{ml} ; \mathrm{t}(43)=2.02, \mathrm{p}<0.05))$. This preference was counterbalanced across diet regime, ensuring that conditioning sessions were working to condition against an innate preference in all diet groups. There was a significant effect of diet on body weight $\left(F_{2,52}=342.87, p<0.001\right)$, with the $F R$ group weighing significantly less than the AL and OB groups and the OB group weighing significantly more than the $A L$ and FR group (Fig 4c) (AL vs OB $t(30)=$ 14.84, $p<3.32 \mathrm{E}-12$; AL vs FR $\mathrm{t}(58)=10.78, \mathrm{p}<1.56 \mathrm{E}-13$; FR vs OB $\mathrm{t}(33)=22.90, \mathrm{p}<1.62 \mathrm{E}-14)$.

There was a significant main effect of CS solution on average consumption during conditioning $\left(F_{1,51}=21.56 p<0.001\right)$ (Fig 4a). There was also a significant interaction between CS solution and diet $\left(F_{3,51}=4.78 p<0.01\right)$. Post hoc analysis revealed that the FR group consumed significantly more $(p<0.001)$, CS solution than the AL, OB and FR-limited diet groups. The FR and FR-limited groups also consumed more CS+ than CS- during conditioning sessions (FR: $t(11)=2.20, p<0.001$; FRlimited $t(10) 2.23 p<0.05)$.

There was a significant interaction between test preference and diet $\left(F_{3,51}=15.09 p<0.001\right)$. Post hoc analysis revealed significant differences between every diet group, with preference decreasing in the order of FR-limited, FR, AL and OB (Fig 4b). The AL $(t(18)=1.73, p<0.001)$, $\mathrm{FR}(\mathrm{t}(10)=1.82, \mathrm{p}<0.01)$ and FR-limited $(\mathrm{t}(9)=1.83, \mathrm{p}<0.001)$ diet groups all acquired significant preferences for the $\mathrm{CS}+$, while the $\mathrm{OB}$ group failed to acquire a significant $\mathrm{CS}+$ preference $(\mathrm{t}(10)=$ $1.82, p=0.37)$.
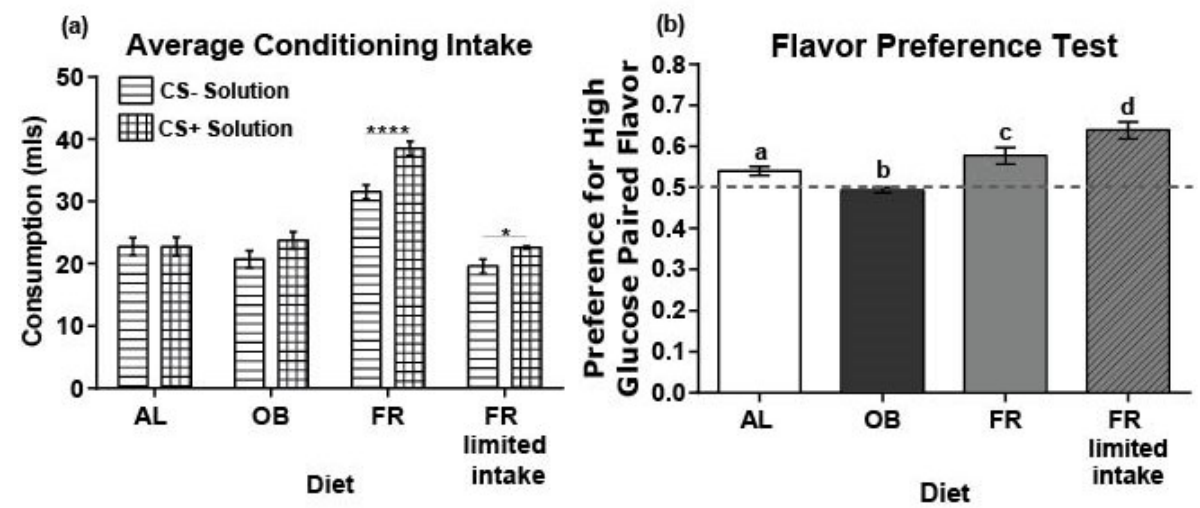

(c)

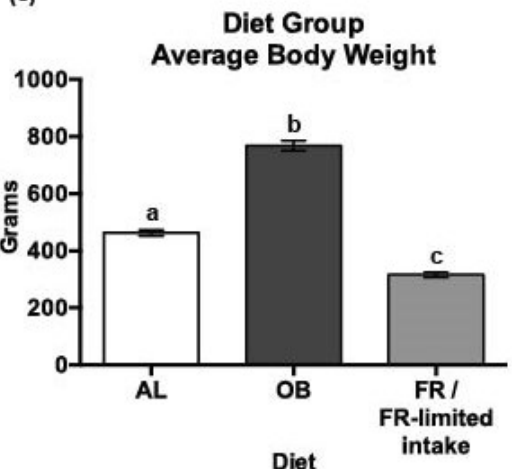

Figure 4 Diet has a significant impact on flavor-nutrient conditioning. (a) Average consumption of high glucose (6.1\% glucose) and low glucose ( $1 \%$ glucose and $0.125 \%$ saccharin solution) conditioning solutions per diet group. (b) Average preference for each diet group for the high glucose paired flavor during a two bottle choice test. Letters (a,b,c,d) indicate a significant difference from each group. (c) Average weight per diet group recorded on test day. Letters (a,b,c) indicate a significant difference from each group. (AL: ad libitum, OB: diet-induced obesity, FR: food restricted, FR-limited intake: food restricted with limited conditioning solution intake).

\subsection{Experiment 2b Results}

During the 7 minute access period, consumption between rats receiving the CS+ and CSconditioning solutions did not differ $(\mathrm{AL} t(8)=2.31, \mathrm{p}=0.73$; FR-limited $\mathrm{t}(7)=2.36 \mathrm{p}=0.22)$. Compared to rats that drank the CS- conditioning solution, rats that consumed the CS+ conditioning 
solution showed significant increases in phosphorylated/total IR Tyr1162/1163 (AL $t(8)=1.89, p$ $<0.05$; FR t(7) $=1.90, \mathrm{p}<0.05)$ (Fig 5a-d).
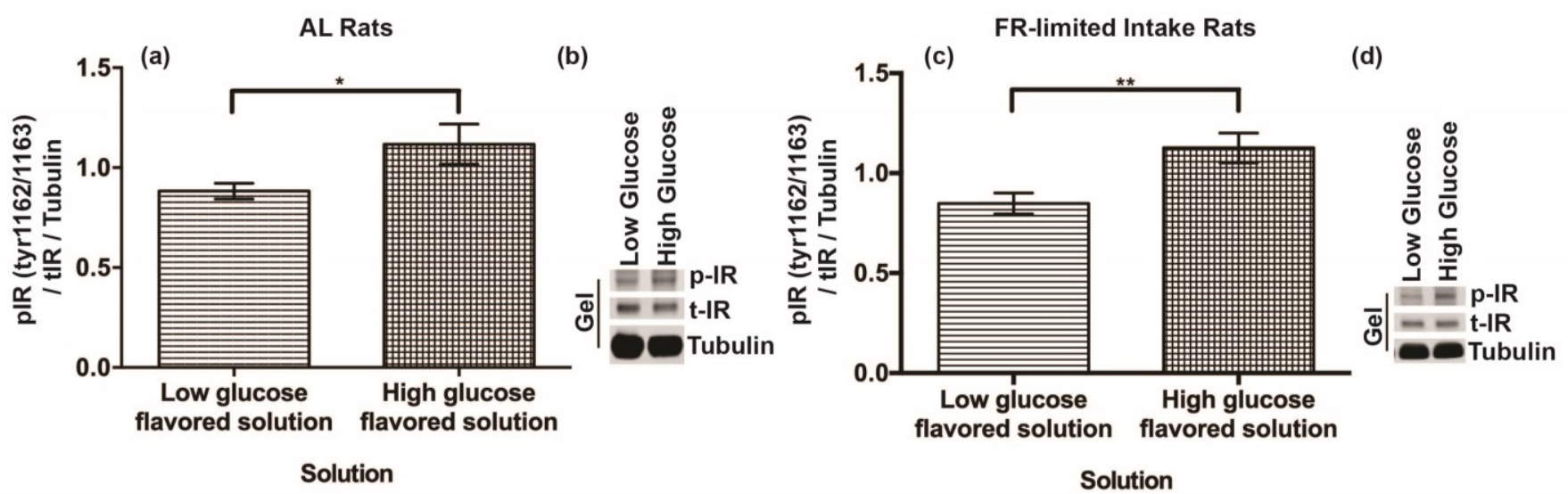

Figure 5 NAc insulin receptor activity reflects consumption across a caloric gradient. NAc insulin receptor phosphorylation at Tyr1162/1163 after 7-minute access to either the low glucose or high glucose conditioning solution. (a,b) Ad libitum fed and (c,d) food restricted-limited intake rats were used from experiment $2 a$.

\subsection{Experiment 2c Results}

Prior to beginning conditioning, the rats showed a baseline preference for the glucose plus saccharin solution $(59 \%, 5 \mathrm{vs} .3 \mathrm{ml} ; \mathrm{t}(16)=2.13, \mathrm{p}<0.001)$. This preference was counterbalanced across microinfusion treatment, ensuring that conditioning sessions were working to condition against an innate preference in both groups.

Analysis of results by a 2x10 mixed ANOVA with repeated measures on CS+ conditioning session indicated a main effect of session $\left(F_{1,15}=18.588, p<.001\right)$ (Fig 6$)$. This reflects rats' increase in consumption throughout conditioning sessions. However, the increase in consumption in the IgG group was higher than that observed in the InsAb group as indicated by the significant interaction between conditioning session and microinfusion treatment $\left(F_{1,15}=2.306, p<.05\right)$. Posthoc analysis revealed significant differences in InsAb versus IgG consumption during the following CS+ conditioning sessions: $6(t(15)=2.651, p<.01), 8(t(15)=2.064, p<.05)$, and $10(t(15)=2.834$, $p<.01)$. During these sessions, rats treated with IgG drank more of the high glucose solution compared to InsAb treated rats, implying that InsAb microinfusions into the NAc shell significantly decreased rats' consumption of the high glucose solution. 
(a) CS+ (High glucose Solution)

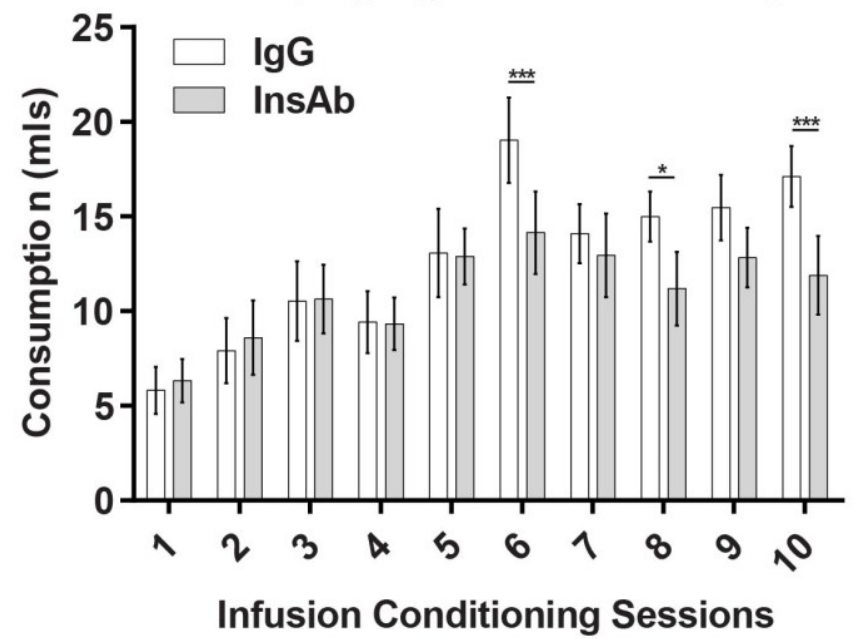

(b)

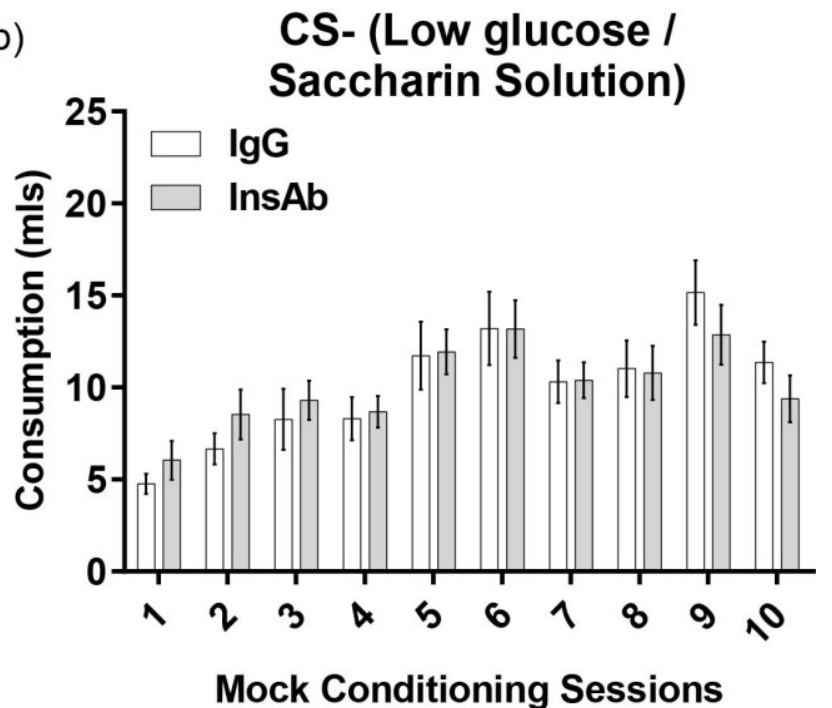

Figure 6 Infusions of InsAb into the NAc shell decreases consumption of a sweet caloric solution. Average consumption (mls) during (a) CS+ and (b) CS- conditioning sessions, in which rats had 30 minute access to the (a) high glucose (6.1\% glucose) flavored conditioning solution or (b) low glucose / saccharin mix (1\% glucose $+0.125 \%$ saccharin) after intracranial infusions of either InsAb or IgG. (InsAb: Insulin antbodies, IgG: immunoglobulin G).

Analysis of results by a 2x10 mixed ANOVA with repeated measures on CS- conditioning session indicated a significant effect of session $\left(F_{1,15}=11.232, p<.001\right)$, but no significant interaction for session and microinfusion treatment $\left(F_{1,15}=0.516, p=.861\right)$. This is expected, as consumption between microinfusion groups should not differ during conditioning sessions preceded by mock microinfusions and consumption of the CS- should increase in both groups as conditioning continues.

There was no significant difference in preference within either microinfusion treatment group between the two tests (4 consecutive overnight sessions) performed after $10 \mathrm{CS}+/ \mathrm{CS}$ - conditioning sessions (IgG: $\mathrm{t}(7)=1.93, \mathrm{p}=0.09$ and $\operatorname{InsAb:t}(8)=1.86, \mathrm{p}=0.56)$; therefore, results of the first and second test sessions were averaged. Analysis of consumption during overnight testing after 10 $\mathrm{CS}+/ \mathrm{CS}$ - conditioning sessions indicated no significant interaction between CS and microinfusion treatment $\left(F_{1,15}=1.46, p=0.246\right)$; there was a significant CS main effect $\left(F_{1,15}=8.15, p<0.05\right)$. Based on Stouffer et al.'s previous finding that InsAb during conditioning with equivalent glucose solutions decreased preference for the paired flavor we pursued the a priori prediction that InsAb group in this experiment would not show preference for the CS+ flavor with a follow-up within group analysis. Evaluation of preference within treatment group showed that the $\lg G$ group significantly preferred the CS+ flavor $(t(7)=3.64, p<0.01)$, while the InsAb group did not $(t(8)=1.03, p=0.33)$ (Fig 7a).

There were no significant differences within either microinfusion treatment group between post conditioning tests (IgG: $F_{2,14}=0.37, p=0.69$ and InsAb: $F_{2,16}=0.23, p=0.79$ ); therefore, all three tests ( 6 consecutive sessions) occurring after the 20 conditioning sessions were averaged together. There was no significant interaction between $C S$ and microinfusion treatment $\left(F_{1,15}=1.72, p=\right.$ $0.209)$. There was a significant main effect of $C S\left(F_{1,15}=6.21, p<0.05\right)$. Evaluation of preference within treatment group showed that the IgG group significantly preferred the CS+ flavor $(t(7)=2.70 p$ $<0.05)$, while the InsAb group did not $(\mathrm{t}(8)=0.84, \mathrm{p}=0.426)$. Overall, overnight testing occurring after both 10 and 20 conditioning sessions produced similar results; rats that received IgG infusions into the NAc shell acquired a significant flavor-nutrient preference, while the InsAb group did not (Fig $7 b)$. 


\section{Overnight Flavor Preference Test}

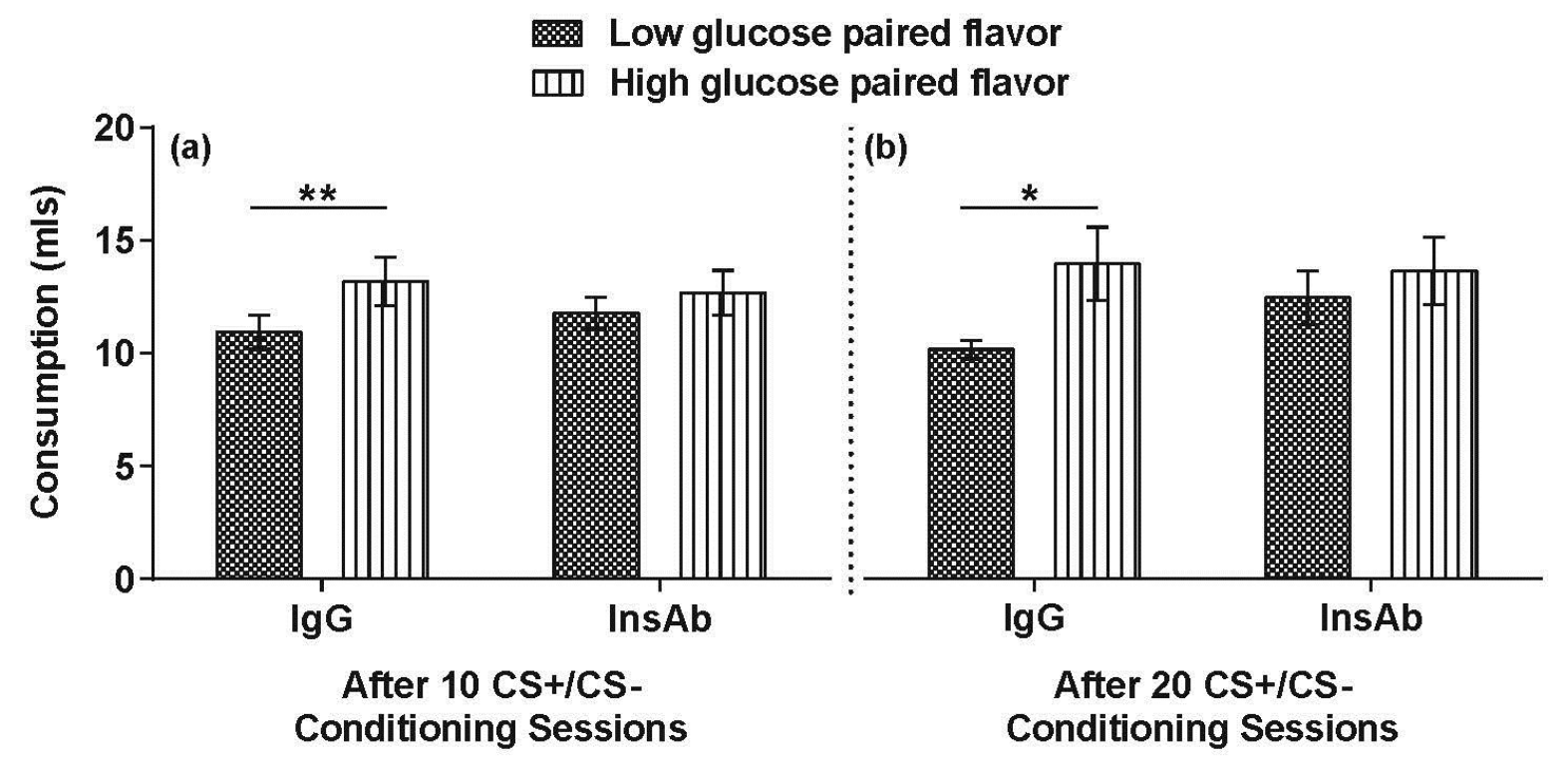

Figure 7 Inhibition of NAc insulin activity during conditioning prevents the formation of a flavor-nutrient preference at test. Average consumption of flavors previously paired with either the low glucose or high glucose conditioning solutions (a) after 10 conditioning sessions and (b) after 20 conditioning sessions.

Although $\lg G$ rats demonstrated a significant preference for the CS+ flavor during overnight testing, the preference was moderate. Therefore, we investigated whether increasing consumption at test, by allowing rats to choose between their conditioning solutions (i.e. both flavor and sweeteners present), would yield larger differences between microinfusion groups. The conditioning solution test was 30 minutes because rats consume a large enough volume of solution to display a preference when sweeteners are present. Analysis revealed a significant main effect of $C S\left(F_{1,15}=5.263, p<\right.$ $0.05)$, but no significant interaction between flavor and microinfusion treatment $\left(F_{1,15}=2.577, p=\right.$ $0.129)$. Similar to findings in the overnight tests, the $\operatorname{lgG}$ group had a significant preference for the $\mathrm{CS}+$ conditioning solution $(\mathrm{t}(7)=1.89, \mathrm{p}<0.01)(\mathrm{Fig} 8)$. Preference for the $\mathrm{IgG}$ group increased from $58 \%$ to $64 \%$ when sweeteners were added back into the test solution. Even when sweeteners were present in the test solutions, the InsAb group failed to display a preference $(t(8)=1.85, p=0.70)$. 


\section{Conditioning Solutions 30 min Preference Test}

Low Glucose

Flavored Solution

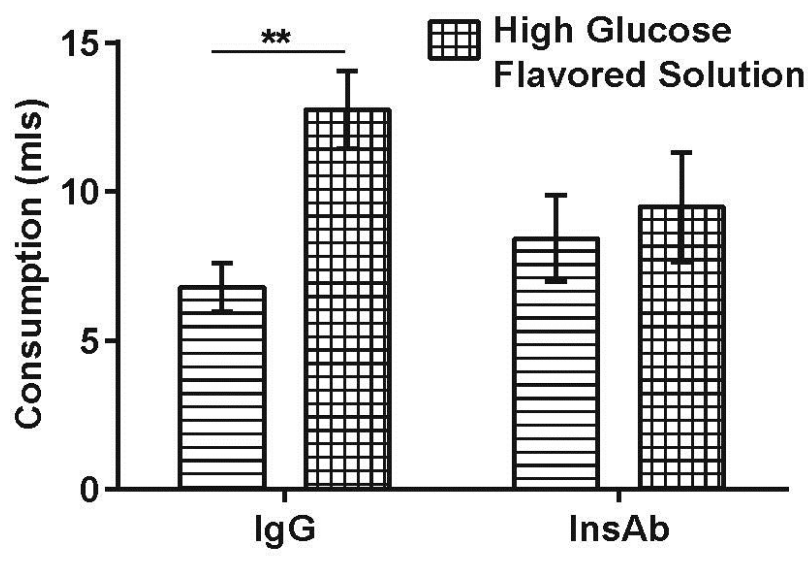

After Flavor Preference Testing Sessions

Figure 8 Inhibition of NAc insulin activity during conditioning prevents the formation of a flavor-nutrient preference at test, even when sweeteners are added into the testing solution. Average consumption of the flavored low glucose (1\% glucose and $0.125 \%$ saccharin) and flavored high glucose $(6.1 \%$ glucose $)$ conditioning solutions after all conditioning and flavor testing sessions.

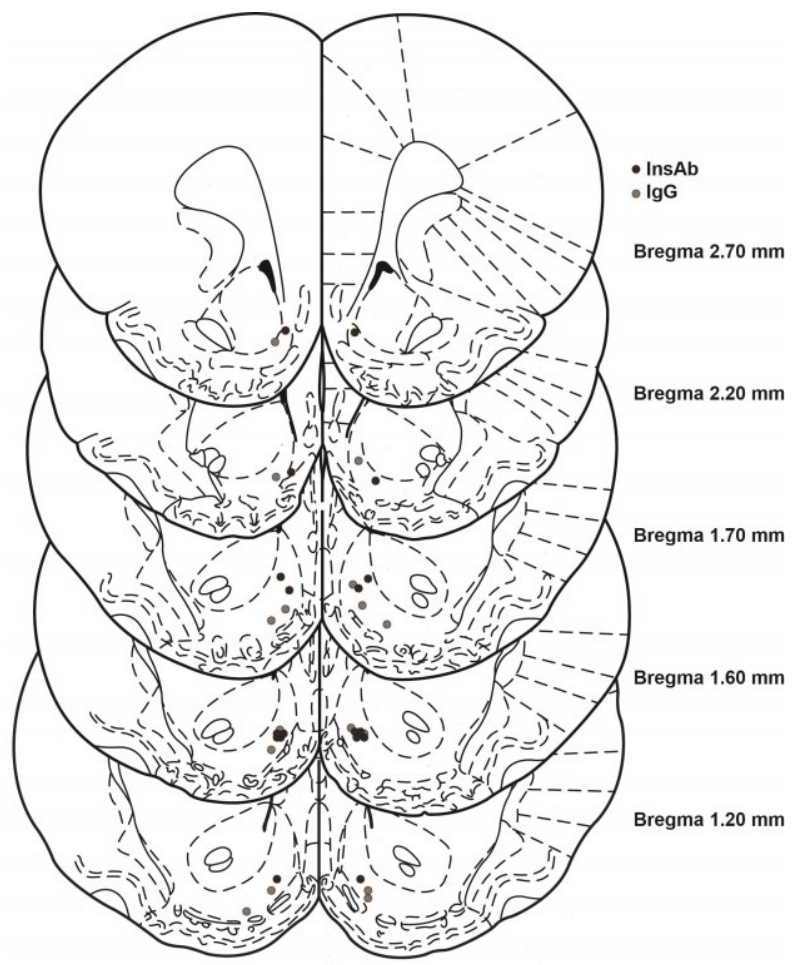

Figure 9 Histologically confirmed cannula placements of rats that received either InsAb or lgG during flavor-nutrient conditioning. (InsAb: Insulin Antibodies, IgG: Immunoglobulin G).

\subsection{Experiment 2d Results}

Similar to observations in AL rats from experiment $1 b$, FR rats with 7 minute access to $16 \%$ glucose displayed significantly more insulin receptor phosphorylation than rats with 7 minute access to water $(\mathrm{t}(8)=3.20, \mathrm{p}<0.01)$. It should also be noted that $\mathrm{FR}$ rats with access to $16 \%$ glucose drank significantly more solution than rats with access to water $(t(8)=6.00 p<0.001)$. NAc shell microinjections of InsAb resulted in significantly less NAc insulin receptor activation than NAc shell 
microinjections of $\operatorname{lgG}(\mathrm{t}(9)=5.0815, \mathrm{p}<0.001)$. Total intake of the $16 \%$ glucose solution did not differ between FR rats receiving NAc shell microinjections of IgG or InsAb $(t(9)=0.37 p=0.72)$.

Nucleus Accumbens Insulin Receptor Phosphorylation in FR rats
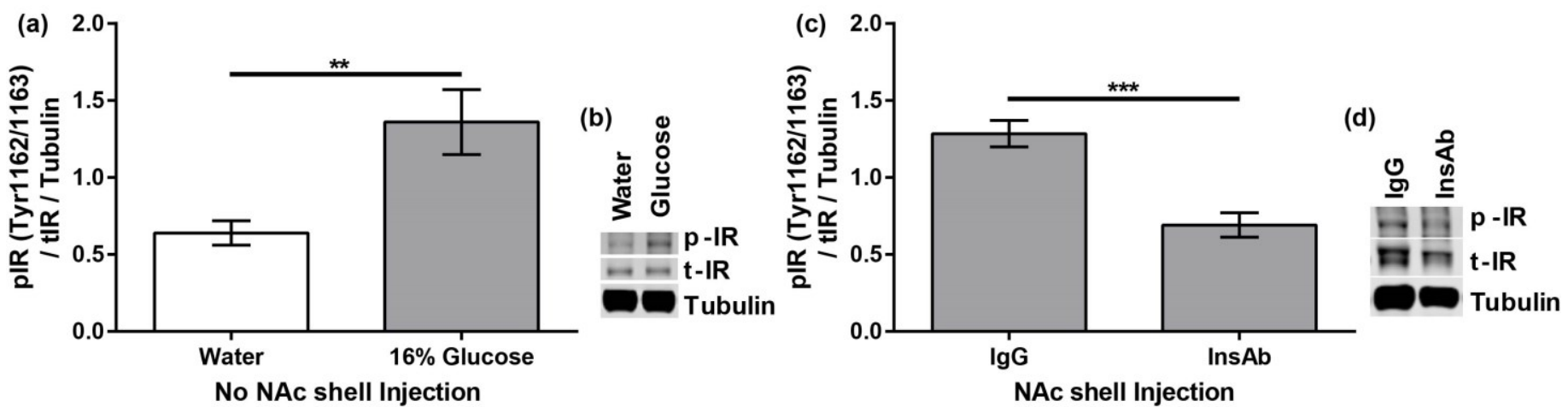

Figure 10 Inhibition of glucose-stimulated NAc insulin receptor activation via InsAb in FR rats. Phosphorylation of the insulin receptor at Tyr1162/1163 in FR rats (a) consuming either water or $16 \%$ glucose or (c) consuming $16 \%$ glucose after NAc shell microinjection of IgG or InsAb. (b,d) representative blots.

\section{Discussion}

In the first series of experiments, we observed significant increases in phosphorylation of the insulin receptor, Akt and GluA1 in the NAc after brief IG infusion or oral consumption of a glucose solution. Glucose is rapidly absorbed and thus may lead to rapid changes in NAc insulin receptor activation that may not be observed if using another food type. These results are in line with early research demonstrating the ability for radiolabeled insulin to cross the blood brain barrier two minutes after intraperitoneal administration (William A. Banks et al., 1997). These results may appear at odds with insulin's typical slow and continual rise in the periphery after the consumption of a carbohydraterich meal. However, the rate of insulin transport even within brain regions vary across time (Banks \& Kastin, 1998). Insulin transport across the blood brain barrier also occurs via the insulin receptor - a saturable method of transport (Banks et al., 2012). Therefore, as peripheral insulin rises in response to consumption of a meal, the rate of trafficking may decrease as the number of available receptors becomes limited.

IG infusions of glucose in all diet groups increased phosphorylation of NAc insulin receptors. However, upon closer examination this increase was not significant in the OB group. The blunted increase observed in the $\mathrm{OB}$ group could be due to decreased transport of insulin across the blood brain barrier, insulin receptor insensitivity in the neural tissue, or both (Banks et al., 2012; Baskin et al., 1985; Kaiyala et al., 2000; Stouffer et al., 2015). Compared to rats that received water, FR and AL glucose receiving rats showed significant increases in NAc insulin receptor activation, with a greater effect in the FR group than the AL group. Though the greater increase observed in the FR group was not significantly different from the $A L$ group, an enhanced response would be expected in low calorie consuming rats that show enhanced trafficking of insulin across the blood brain barrier (W. A. Banks et al., 1997; Urayama \& Banks, 2008). The diet dependent responses are in accordance with previous imaging studies showing that insulin resistant subjects show decreased insulin-induced activation in the ventral striatum (Anthony et al., 2006) and insulin sensitivity correlates with D2 DA receptor availability (Dunn et al., 2012).

These findings have important implications for today's Western societies where obesity rates are on the rise and high calorie foods are readily available (Stice et al., 2013). Consumption is modulated by hypothalamic regions that monitor energy homeostasis, as well as the mesolimbic dopamine pathway which registers the rewarding and reinforcing aspects of palatable food intake. While the etiology of obesity is heterogeneous, many suggest that the excessive food consumption can be driven by the need to satisfy a hyposensitive brain reward circuit (Kenny, 2011; Stice et al., 2010; Volkow et al., 2003). Insulin's ability to induce increased extracellular dopamine release in the 
striatum and reflect glycemic intake via insulin receptor activation is impaired in an OB diet model, suggesting that obese individuals may have difficulty accurately assessing the nutritive value of food (Stouffer et al., 2015). Our results further support the idea that neural insulin signaling is impaired in OB diet models, which can lead to altered striatal signaling and learning about food nutritive value. Obese individuals may overeat to obtain sufficient levels of striatal activation, leading to a diet that stimulates insulin insensitivity and thus may further exacerbate a hyposensitive reward system.

In the second series of experiments, we tested the hypothesis that insulin activity in the NAc shell plays a role in the formation of flavor-nutrient preferences. Dopamine signaling in the ventral striatum is necessary for acquiring a flavor-nutrient preference (Touzani et al., 2008). Insulin also mediates flavor preferences and enhances extracellular evoked dopamine release in the NAc; further, the dopamine-enhancing effect is greater in FR rats, but blunted in OB rats (Stouffer et al., 2015). Now, we observed that diet had a significant impact on the ability to form a flavor-nutrient preference; $\mathrm{AL}$ and $\mathrm{FR}$ rats acquired significant flavor-nutrient preferences, while $\mathrm{OB}$ rats did not. Limiting conditioning solution intake in FR rats to match the average consumption of AL controls, only enhanced preference at test. Flavor-nutrient preferences within diet group mirror the insulin-induced enhancement of striatal dopamine release - enhanced in FR, but blunted in OB. Although the parallel between phosphorylation of insulin receptor and GluA1 does not necessarily imply a causal relation, it at least suggests concurrent insulin signaling and DA release; moreover, the established role of GluA1 phosphorylation in synaptic plasticity (Kessels \& Malinow, 2009) points to a possible mechanistic underpinning of flavor-nutrient learning. Taken together with findings from the first series of experiments, the impaired insulin receptor activation observed in the OB group likely results in the impaired ability to assign proper nutritive value to the CS+ flavor. Thus obese individuals, with insulin insensitivity, may make food choices based on inaccurate nutritive assessments. Such faulty evaluations could mean the overconsumption of food or the selection of foods that don't accurately meet the body's current physiological needs.

Enhanced flavor-nutrient preference has been reported in FR rodents; however, some studies find no difference in preference between ad lib and food restricted animals. These studies vary on a variety of factors, including duration of diet manipulation, method of nutrient delivery, species and sex (Ackroff \& Sclafani, 2015; Capaldi et al., 1994; Fedorchak \& Bolles, 1987; Yiin et al., 2005b).

Flavor-nutrient preference in diet-induced-obesity models is less clear. In contrast to the present results, Wald et al., observed enhanced flavor-nutrient preference in OB rats receiving IG infusions of $12 \%$ glucose paired with oral consumption of a CS+ flavored saccharin solution (Wald \& Myers, 2015). These rats were maintained on a cafeteria diet, which allows for exposure to a wide variety of foods. The differences in the two flavor-nutrient paradigms and diet models of obesity may account for the different findings. There is increasing evidence that OB rats show decreased sensitivity to low concentrations of sweeteners and an enhanced preference for higher concentration of sweeteners (Duca et al., 2014; Robinson et al., 2015; Shin et al., 2011). Thus it may be easier for an $\mathrm{OB}$ rat to distinguish between water and $12 \%$ glucose, than $6.1 \%$ glucose and a mixture of $1 \%$ glucose and $0.125 \%$ Saccharin. Alternatively, OB rats may learn the difference in nutritive value between the two solutions, but still prefer consumption of the sweeter, less caloric solution - or the solution for which there was a greater unconditioned preference, presumably based on palatability. This rationale would be consistent with studies demonstrating that various models of diet-inducedobesity result in rats that are less motivated to obtain low sweetened food reward, an effect reversed by fasting (Davis et al., 2008; Narayanaswami et al., 2013). Varying methodology used for dietinduced-obesity, such as a cafeteria diet or unlimited access to one fattening food source, may also have a significant impact on food preferences. Rats kept on a limited high fat diet, thus maintaining healthy weight while consuming unhealthy foods, still show a decreased responding for sucrose reward (Davis et al., 2008). Thus neurochemical or behavioral changes may occur from exposure to a variety of foods used to induce weight gain and not just weight gain alone. 
Adding to the finding that NAc insulin activity reflects consumption of a glucose solution, NAc insulin receptor phosphorylation levels also differed in rats consuming glucose solutions with different sugar concentrations. Thus insulin receptor activation in the NAc is not an 'all or none' effect and may provide more information about the amount of sugar consumed.

Pharmacological manipulation of insulin during flavor-nutrient conditioning yielded observations similar to those in our prior study (Stouffer et al., 2015); infusions of InsAb into the NAc shell decreased consumption of a sweet caloric solution. Even though these findings are at odds with studies showing that inhibition or enhancement of insulin activity in the hypothalamus drives or reduces feeding respectively (Strubbe \& Mein, 1977), these results are in line with the observation that NAc infusions of insulin increased both sucrose intake and the duration in which rats would respond for sucrose reward (Figlewicz et al., 2008). Thus, behavioral consequences of insulin action are likely dependent on brain region and the aspect of ingestive behavior being regulated.

While the IgG group acquired a significant preference for the CS+ flavor associated with the more concentrated sugar solution, the InsAb group did not. Even when sweeteners were added back into the test solutions, only the IgG group demonstrated a significant preference for the CS+ solution. The lack of flavor-nutrient preference in the InsAb group in the test sessions was likely not due to decreased consumption of the CS+ solution during conditioning, as limiting consumption of conditioning solution in FR rats in experiment 1 enhanced rather than reduced their preference. These findings are consistent with previous results, demonstrating a weakened flavor-nutrient preference in rats with impaired insulin signaling throughout the body (Ackroff et al., 1997).

The present results also fall in line with recent imaging studies highlighting a relationship between striatal dopamine and insulin. In healthy normal weight individuals, D2 receptor binding has a positive correlation with insulin sensitivity and a negative correlation with total insulin levels (Caravaggio et al., 2015). But changes in diet seem to alter this relationship, as insulin activity is directly related to body mass index (Wang et al., 2014). Consumption of glucose increases dopamine in the ventral striatum of normal subjects, but decreases dopamine in the ventral striatum of obese subjects.

Given the importance of food consumption for survival across species, it would not be surprising to find that many mechanisms act in parallel to reinforce the consumption of nutritious foods (de Araujo et al., 2012; Fernandes et al., 2013). Multiple studies highlight a role for various metabolic peptides in food reward, including ghrelin, (Abizaid et al., 2006; Malik et al., 2008) leptin (Davis et al., 2011; Figlewicz et al., 2004; Krügel et al., 2003), cholecystokinin (Hajnal et al., 2007), and glucagon-like peptide 1 (Dickson et al., 2012; Richard et al., 2015). Similarly, macronutrients such as glucose, amino acids and fatty acids, may modulate food reward (Fernandes et al., 2013). In naïve mice, consumption of glucose stimulates increases in extracellular dopamine, and this increase is blocked by the competing non-nutritive glucose analogue 2-deoxy-D-glucose (2-DG) (Tellez et al., 2013). Tellez et al. hypothesized that 2-DG's ability to inhibit glucose-induced dopamine release may be due to interference in insulin signaling. Our results are consistent with this idea, suggesting that impaired insulin signaling, associated with impaired striatal dopamine release, may lead to inaccurate valuation of nutritive foods.

Taken together, the present findings suggest that insulin in the NAc may act as one of the signals communicating the value of nutritive solutions - rapidly detecting the consumption of a caloric solution and playing a role in the acquisition and updating of food preferences.

\section{Acknowledgements}

The research was supported by DA033811, a grant from the NIDA/NIH (K.D.C.) and the NSF Graduate Research Fellowship program (C.A.W.). We would also like to thank Kwame McCartney for his technical assistance and support.

\section{References}


Abizaid, A., Liu, Z.-W., Andrews, Z. B., Shanabrough, M., Borok, E., Elsworth, J. D., ... Tschöp, M. H. (2006). Ghrelin modulates the activity and synaptic input organization of midbrain dopamine neurons while promoting appetite. Journal of Clinical Investigation, 116(12), 3229.

Ackroff, K., \& Sclafani, A. (2015). Flavor change and food deprivation are not critical for post-oral glucose appetition in mice. Physiol Behav, 140, 23-31.

Ackroff, K., Sclafani, A., \& Axen, K. V. (1997). Diabetic rats prefer glucose-paired flavors over fructosepaired flavors. Appetite, 28(1), 73-83. doi: 10.1006/appe.1996.0058

Ackroff, K., Yiin, Y. M., \& Sclafani, A. (2010). Post-oral infusion sites that support glucose-conditioned flavor preferences in rats. Physiol Behav, 99(3), 402-411. doi: 10.1016/j.physbeh.2009.12.012

Anthony, K., Reed, L. J., Dunn, J. T., Bingham, E., Hopkins, D., Marsden, P. K., \& Amiel, S. A. (2006). Attenuation of insulin-evoked responses in brain networks controlling appetite and reward in insulin resistance: the cerebral basis for impaired control of food intake in metabolic syndrome? Diabetes, 55(11), 2986-2992. doi: 10.2337/db06-0376

Banke, T., Bowie, D., Lee, H.-K., Huganir, R., Schousboe, A., \& Traynelis, S. (2000). Control of GluR1 AMPA receptor function by cAMP-dependent protein kinase. The Journal of neuroscience, 20(1), 89-102.

Banks, W. A., Jaspan, J. B., Huang, W., \& Kastin, A. J. (1997). Transport of Insulin Across the Blood-Brain Barrier: Saturability at Euglycemic Doses of Insulin. Peptides, 18(9), 1423-1429. doi: http://dx.doi.org/10.1016/S0196-9781(97)00231-3

Banks, W. A., Jaspan, J. B., \& Kastin, A. J. (1997). Effect of diabetes mellitus on the permeability of the blood-brain barrier to insulin. Peptides, 18(10), 1577-1584.

Banks, W. A., \& Kastin, A. J. (1998). Differential Permeability of the Blood-Brain Barrier to Two Pancreatic Peptides: Insulin and Amylin. Peptides, 19(5), 883-889. doi: http://dx.doi.org/10.1016/S01969781(98)00018-7

Banks, W. A., Owen, J. B., \& Erickson, M. A. (2012). Insulin in the brain: there and back again. Pharmacol Ther, 136(1), 82-93. doi: 10.1016/j.pharmthera.2012.07.006

Baskin, D. G., Stein, L. J., Ikeda, H., Woods, S. C., Figlewicz, D. P., Porte, D., Jr., . . Dorsa, D. M. (1985). Genetically obese Zucker rats have abnormally low brain insulin content. Life Sci, 36(7), 627-633.

Bernal, S. Y., Dostova, I., Kest, A., Abayev, Y., Kandova, E., Touzani, K., . . Bodnar, R. J. (2008). Role of dopamine D1 and D2 receptors in the nucleus accumbens shell on the acquisition and expression of fructose-conditioned flavor-flavor preferences in rats. Behav Brain Res, 190(1), 59-66. doi: 10.1016/j.bbr.2008.02.003

Capaldi, E. D., Owens, J., \& Palmer, K. A. (1994). Effects of food deprivation on learning and expression of flavor preferences conditioned by saccharin or sucrose. Animal Learning \& Behavior, 22(2), 173180.

Caravaggio, F., Borlido, C., Hahn, M., Feng, Z., Fervaha, G., Gerretsen, P., . . Graff-Guerrero, A. (2015). Reduced insulin sensitivity is related to less endogenous dopamine at D2/3 receptors in the ventral striatum of healthy nonobese humans. Int J Neuropsychopharmacol, 18(7), pyv014. doi: 10.1093/ijnp/pyv014

Carr, K. D. (2011). Food scarcity, neuroadaptations, and the pathogenic potential of dieting in an unnatural ecology: binge eating and drug abuse. Physiol Behav, 104(1), 162-167. doi: 10.1016/j.physbeh.2011.04.023

Carr, K. D., Chau, L. S., Cabeza de Vaca, S., Gustafson, K., Stouffer, M., Tukey, D. S., ... Ziff, E. B. (2010). AMPA receptor subunit GluR1 downstream of D-1 dopamine receptor stimulation in nucleus accumbens shell mediates increased drug reward magnitude in food-restricted rats. Neuroscience, 165(4), 1074-1086. doi: 10.1016/j.neuroscience.2009.11.015

Davis, J. F., Choi, D. L., Schurdak, J. D., Fitzgerald, M. F., Clegg, D. J., Lipton, J. W., . . Benoit, S. C. (2011). Leptin regulates energy balance and motivation through action at distinct neural circuits. Biol Psychiatry, 69(7), 668-674. 
Davis, J. F., Tracy, A. L., Schurdak, J. D., Tschop, M. H., Lipton, J. W., Clegg, D. J., \& Benoit, S. C. (2008). Exposure to elevated levels of dietary fat attenuates psychostimulant reward and mesolimbic dopamine turnover in the rat. Behav Neurosci, 122(6), 1257-1263. doi: 10.1037/a0013111

de Araujo, I. E., Ferreira, J. G., Tellez, L. A., Ren, X., \& Yeckel, C. W. (2012). The gut-brain dopamine axis: a regulatory system for caloric intake. Physiol Behav, 106(3), 394-399. doi:

10.1016/j.physbeh.2012.02.026

Dickson, S. L., Shirazi, R. H., Hansson, C., Bergquist, F., Nissbrandt, H., \& Skibicka, K. P. (2012). The glucagon-like peptide 1 (GLP-1) analogue, exendin-4, decreases the rewarding value of food: a new role for mesolimbic GLP-1 receptors. The Journal of Neuroscience, 32(14), 4812-4820.

Duca, F. A., Swartz, T. D., \& Covasa, M. (2014). Effect of Diet on Preference and Intake of Sucrose in Obese Prone and Resistant Rats. PLoS One, 9(10), e111232. doi: 10.1371/journal.pone.0111232

Dunn, J. P., Kessler, R. M., Feurer, I. D., Volkow, N. D., Patterson, B. W., Ansari, M. S., ... Abumrad, N. N. (2012). Relationship of dopamine type 2 receptor binding potential with fasting neuroendocrine hormones and insulin sensitivity in human obesity. Diabetes care, 35(5), 1105-1111.

Fedorchak, P. M., \& Bolles, R. C. (1987). Hunger enhances the expression of calorie- but not tastemediated conditioned flavor preferences. J Exp Psychol Anim Behav Process, 13(1), 73-79.

Fernandes, M. F., Sharma, S., Hryhorczuk, C., Auguste, S., \& Fulton, S. (2013). Nutritional Controls of Food Reward. Canadian journal of diabetes, 37(4), 260-268.

Fernstrom, J. D., Munger, S. D., Sclafani, A., de Araujo, I. E., Roberts, A., \& Molinary, S. (2012). Mechanisms for sweetness. The Journal of nutrition, 142(6), 1134S-1141S.

Figlewicz, D. P., Bennett, J., Evans, S. B., Kaiyala, K., Sipols, A. J., \& Benoit, S. C. (2004). Intraventricular insulin and leptin reverse place preference conditioned with high-fat diet in rats. Behav Neurosci, 118(3), 479-487. doi: 10.1037/0735-7044.118.3.479

Figlewicz, D. P., Bennett, J. L., Aliakbari, S., Zavosh, A., \& Sipols, A. J. (2008). Insulin acts at different CNS sites to decrease acute sucrose intake and sucrose self-administration in rats. Am J Physiol Regul Integr Comp Physiol, 295(2), R388-394. doi: 10.1152/ajpregu.90334.2008

Geiger, B. M., Haburcak, M., Avena, N. M., Moyer, M. C., Hoebel, B. G., \& Pothos, E. N. (2009). Deficits of mesolimbic dopamine neurotransmission in rat dietary obesity. Neuroscience, 159(4), 1193-1199. doi: 10.1016/j.neuroscience.2009.02.007

Hajnal, A., Acharya, N. K., Grigson, P. S., Covasa, M., \& Twining, R. C. (2007). Obese OLETF rats exhibit increased operant performance for palatable sucrose solutions and differential sensitivity to D2 receptor antagonism. American Journal of Physiology-Regulatory, Integrative and Comparative Physiology, 293(5), R1846-R1854.

Jones, D. M., Arters, J., \& Berger-Sweeney, J. (1999). Carbon dioxide-induced anesthesia has no effect on brain biogenic amine concentrations in mice. Comparative Medicine, 49(3), 316-318.

Kaiyala, K. J., Prigeon, R. L., Kahn, S. E., Woods, S. C., \& Schwartz, M. W. (2000). Obesity induced by a highfat diet is associated with reduced brain insulin transport in dogs. Diabetes, 49(9), 1525-1533.

Kenny, P. J. (2011). Reward mechanisms in obesity: new insights and future directions. Neuron, 69(4), 664-679.

Kessels, H. W., \& Malinow, R. (2009). Synaptic AMPA receptor plasticity and behavior. Neuron, 61(3), 340350.

Krügel, U., Schraft, T., Kittner, H., Kiess, W., \& Illes, P. (2003). Basal and feeding-evoked dopamine release in the rat nucleus accumbens is depressed by leptin. Eur J Pharmacol, 482(1), 185-187.

Malik, S., McGlone, F., Bedrossian, D., \& Dagher, A. (2008). Ghrelin modulates brain activity in areas that control appetitive behavior. Cell Metabolism, 7(5), 400-409.

McCaleb, M., \& Myers, R. (1979). Striatal dopamine release is altered by glucose and insulin during pushpull perfusion of the rat's caudate nucleus. Brain research bulletin, 4(5), 651-656. 
McNay, E. C., Ong, C. T., McCrimmon, R. J., Cresswell, J., Bogan, J. S., \& Sherwin, R. S. (2010). Hippocampal memory processes are modulated by insulin and high-fat-induced insulin resistance. Neurobiology of learning and memory, 93(4), 546-553.

Narayanaswami, V., Thompson, A., Cassis, L., Bardo, M., \& Dwoskin, L. (2013). Diet-induced obesity: dopamine transporter function, impulsivity and motivation. Int J Obes, 37(8), 1095-1103.

Owens, W. A., Sevak, R. J., Galici, R., Chang, X., Javors, M. A., Galli, A., . . . Daws, L. C. (2005). Deficits in dopamine clearance and locomotion in hypoinsulinemic rats unmask novel modulation of dopamine transporters by amphetamine. J Neurochem, 94(5), 1402-1410. doi: 10.1111/j.14714159.2005.03289.x

Paranjape, S. A., Chan, O., Zhu, W., Horblitt, A. M., McNay, E. C., Cresswell, J. A., . . Sherwin, R. S. (2010). Influence of insulin in the ventromedial hypothalamus on pancreatic glucagon secretion in vivo. Diabetes, 59(6), 1521-1527. doi: 10.2337/db10-0014

Richard, J. E., Anderberg, R. H., Göteson, A., Gribble, F. M., Reimann, F., \& Skibicka, K. P. (2015). Activation of the GLP-1 Receptors in the Nucleus of the Solitary Tract Reduces Food Reward Behavior and Targets the Mesolimbic System.

Robinson, M. J., Burghardt, P. R., Patterson, C. M., Nobile, C. W., Akil, H., Watson, S. J., ... Ferrario, C. R. (2015). Individual Differences in Cue-Induced Motivation and Striatal Systems in Rats Susceptible to Diet-Induced Obesity. Neuropsychopharmacology, 40(9), 2113-2123. doi: 10.1038/npp.2015.71

Roche, K. W., O'Brien, R. J., Mammen, A. L., Bernhardt, J., \& Huganir, R. L. (1996). Characterization of multiple phosphorylation sites on the AMPA receptor GluR1 subunit. Neuron, 16(6), 1179-1188.

Schulingkamp, R. J., Pagano, T. C., Hung, D., \& Raffa, R. B. (2000). Insulin receptors and insulin action in the brain: review and clinical implications. Neuroscience \& Biobehavioral Reviews, 24(8), 855-872. doi: http://dx.doi.org/10.1016/S0149-7634(00)00040-3

Shin, A. C., Townsend, R. L., Patterson, L. M., \& Berthoud, H. R. (2011). "Liking" and "wanting" of sweet and oily food stimuli as affected by high-fat diet-induced obesity, weight loss, leptin, and genetic predisposition. Am J Physiol Regul Integr Comp Physiol, 301(5), R1267-1280. doi:

10.1152/ajpregu.00314.2011

Snyder, G. L., Allen, P. B., Fienberg, A. A., Valle, C. G., Huganir, R. L., Nairn, A. C., \& Greengard, P. (2000). Regulation of phosphorylation of the GluR1 AMPA receptor in the neostriatum by dopamine and psychostimulants in vivo. The Journal of neuroscience, 20(12), 4480-4488.

Stice, E., Figlewicz, D. P., Gosnell, B. A., Levine, A. S., \& Pratt, W. E. (2013). The contribution of brain reward circuits to the obesity epidemic. Neurosci Biobehav Rev, 37(9 Pt A), 2047-2058. doi: 10.1016/j.neubiorev.2012.12.001

Stice, E., Yokum, S., Blum, K., \& Bohon, C. (2010). Weight gain is associated with reduced striatal response to palatable food. J Neurosci, 30(39), 13105-13109. doi: 10.1523/jneurosci.2105-10.2010

Stouffer, M. A., Woods, C. A., Patel, J. C., Lee, C. R., Witkovsky, P., Bao, L., . . Rice, M. E. (2015). Insulin enhances striatal dopamine release by activating cholinergic interneurons and thereby signals reward. Nat Commun, 6. doi: 10.1038/ncomms9543

Strubbe, J. H., \& Mein, C. G. (1977). Increased feeding in response to bilateral injection of insulin antibodies in the VMH. Physiol Behav, 19(2), 309-313.

Tellez, L. A., Ren, X., Han, W., Medina, S., Ferreira, J. G., Yeckel, C. W., \& de Araujo, I. E. (2013). Glucose utilization rates regulate intake levels of artificial sweeteners. J Physiol, 591(Pt 22), 5727-5744. doi: 10.1113/jphysiol.2013.263103

Touzani, K., Bodnar, R., \& Sclafani, A. (2008). Activation of dopamine D1-like receptors in nucleus accumbens is critical for the acquisition, but not the expression, of nutrient-conditioned flavor preferences in rats. Eur J Neurosci, 27(6), 1525-1533. doi: 10.1111/j.1460-9568.2008.06127.x

Urayama, A., \& Banks, W. A. (2008). Starvation and triglycerides reverse the obesity-induced impairment of insulin transport at the blood-brain barrier. Endocrinology, 149(7), 3592-3597. doi:

10.1210/en.2008-0008 
Volkow, N. D., Wang, G. J., Maynard, L., Jayne, M., Fowler, J. S., Zhu, W., ... Pappas, N. (2003). Brain dopamine is associated with eating behaviors in humans. Int J Eat Disord, 33(2), 136-142. doi: 10.1002/eat.10118

Wald, H. S., \& Myers, K. P. (2015). Enhanced flavor-nutrient conditioning in obese rats on a high-fat, highcarbohydrate choice diet. Physiol Behav, 151, 102-110. doi: 10.1016/j.physbeh.2015.07.002

Wang, G. J., Tomasi, D., Convit, A., Logan, J., Wong, C. T., Shumay, E., ... Volkow, N. D. (2014). BMI modulates calorie-dependent dopamine changes in accumbens from glucose intake. PLoS One, 9(7), e101585. doi: 10.1371/journal.pone.0101585

Warwick, Z. S., \& Weingarten, H. P. (1994). Dissociation of palatability and calorie effects in learned flavor preferences. Physiol Behav, 55(3), 501-504.

Williams, J. M., Owens, W. A., Turner, G. H., Saunders, C., Dipace, C., Blakely, R. D., . . Galli, A. (2007). Hypoinsulinemia regulates amphetamine-induced reverse transport of dopamine. PLoS Biol, 5(10), e274. doi: 10.1371/journal.pbio.0050274

Yiin, Y. M., Ackroff, K., \& Sclafani, A. (2005a). Flavor preferences conditioned by intragastric nutrient infusions in food restricted and free-feeding rats. Physiol Behav, 84(2), 217-231. doi: 10.1016/j.physbeh.2004.11.008

Yiin, Y. M., Ackroff, K., \& Sclafani, A. (2005b). Food deprivation enhances the expression but not acquisition of flavor acceptance conditioning in rats. Appetite, 45(2), 152-160. doi: 10.1016/j.appet.2005.04.002

Zhen, J., Reith, M. E., \& Carr, K. D. (2006). Chronic food restriction and dopamine transporter function in rat striatum. Brain Res, 1082(1), 98-101. doi: 10.1016/j.brainres.2006.01.094 